\title{
Clinical Categorization Algorithm (CLICAL) and Machine Learning Approach (SRF-CLICAL) to Predict Clinical Benefit to Immunotherapy in Metastatic Melanoma Patients: Real-World Evidence from the Istituto Nazionale Tumori IRCCS Fondazione Pascale, Napoli, Italy
}

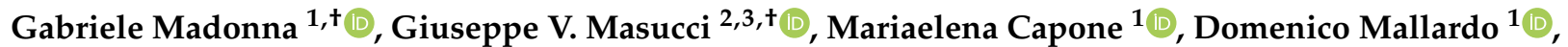 \\ Antonio Maria Grimaldi ${ }^{1}$, Ester Simeone ${ }^{1}$, Vito Vanella ${ }^{1}$, Lucia Festino ${ }^{1}$, Marco Palla ${ }^{1}$, Luigi Scarpato ${ }^{1}$, \\ Marilena Tuffanelli ${ }^{1}$, Grazia D'angelo ${ }^{1}$, Lisa Villabona ${ }^{2}$ D, Isabelle Krakowski ${ }^{3,4}$, Hanna Eriksson ${ }^{2,3}$, \\ Felipe Simao ${ }^{5}$, Rolf Lewensohn ${ }^{2,3}$ and Paolo Antonio Ascierto ${ }^{1, *(D)}$
}

\section{check for}

updates

Citation: Madonna, G.; Masucci, G.V.; Capone, M.; Mallardo, D.; Grimaldi, A.M.; Simeone, E.; Vanella, V.; Festino, L.; Palla, M.; Scarpato, L.; et al. Clinical Categorization

Algorithm (CLICAL) and Machine Learning Approach (SRF-CLICAL) to Predict Clinical Benefit to

Immunotherapy in Metastatic

Melanoma Patients: Real-World

Evidence from the Istituto Nazionale Tumori IRCCS Fondazione Pascale, Napoli, Italy. Cancers 2021, 13, 4164 https://doi.org/10.3390/

cancers13164164

Academic Editor: Sandeep K. Singhal

Received: 5 July 2021

Accepted: 16 August 2021

Published: 19 August 2021

Publisher's Note: MDPI stays neutral with regard to jurisdictional claims in published maps and institutional affiliations.

Copyright: (c) 2021 by the authors. Licensee MDPI, Basel, Switzerland. This article is an open access article distributed under the terms and conditions of the Creative Commons Attribution (CC BY) license (https:/ / creativecommons.org/licenses/by/ $4.0 /)$.
1 Melanoma, Cancer Immunotherapy and Development Therapeutics Unit, Istituto Nazionale Tumori IRCCS Fondazione G. Pascale, 80131 Napoli, Italy; g.madonna@istitutotumori.na.it (G.M.); me.capone@istitutotumori.na.it (M.C.); d.mallardo@istitutotumori.na.it (D.M.); a.grimaldi@istitutotumori.na.it (A.M.G.); e.simeone@istitutotumori.na.it (E.S.); v.vanella@istitutotumori.na.it (V.V.); 1.festino@istitutotumori.na.it (L.F.); m.palla@istitutotumori.na.it (M.P.); 1.scarpato@istitutotumori.na.it (L.S.); m.tuffanelli@istitutotumori.na.it (M.T.); grazia.dangelo@istitutotumori.na.it (G.D.)

2 Theme Cancer, Karolinska University Hospital, 17176 Stockholm, Sweden; giuseppe.masucci@ki.se (G.V.M.); lisa.villabona@ki.se (L.V.); hanna.eriksson@sll.se (H.E.); rolf.lewensohn@ki.se (R.L.)

3 Department of Oncology and Pathology, Karolinska Institutet, 17164 Stockholm, Sweden; isabelle.krakowski@ki.se

4 Theme Inflammation, Karolinska University Hospital, 17176 Stockholm, Sweden

5 Genevia Technologies OY, 33100 Tampere, Finland; felipe.simao@geneviatechnologies.com

* Correspondence: p.ascierto@istitutotumori.na.it

$+\quad$ These authors equally contributed.

Simple Summary: Immune checkpoint inhibitors have improved the prognosis for patients with advanced melanoma. Despite the recent success of immunotherapy, many patients still do not benefit from these treatments, and their real-life application may yield different outcomes compared to the advantage presented in clinical trials. There is therefore a need to select patients who can really benefit from these treatments. We have focused our study on a real-life retrospective analysis of metastatic melanoma patients treated with immunotherapy at a single institution-the Istituto Nazionale Tumori IRCCS Fondazione "G. Pascale" of Napoli, Italy. With the help of AI and machine learning we validated an algorithm based on clinical variables of patients-namely, the Clinical Categorization Algorithm (CLICAL) - that defines five predictable cohorts of benefit to immunotherapy with 95\% accuracy. It can be a useful tool for the stratification of metastatic melanoma patients who may or may not improve from immunotherapy treatment.

Abstract: The real-life application of immune checkpoint inhibitors (ICIs) may yield different outcomes compared to the benefit presented in clinical trials. For this reason, there is a need to define the group of patients that may benefit from treatment. We retrospectively investigated 578 metastatic melanoma patients treated with ICIs at the Istituto Nazionale Tumori IRCCS Fondazione "G. Pascale" of Napoli, Italy (INT-NA). To compare patients' clinical variables (i.e., age, lactate dehydrogenase (LDH), neutrophil-lymphocyte ratio (NLR), eosinophil, BRAF status, previous treatment) and their predictive and prognostic power in a comprehensive, non-hierarchical manner, a clinical categorization algorithm (CLICAL) was defined and validated by the application of a machine learning algorithm-survival random forest (SRF-CLICAL). The comprehensive analysis of the clinical parameters by log risk-based algorithms resulted in predictive signatures that could identify groups of patients with great benefit or not, regardless of the ICI received. From a real-life retrospective analysis of metastatic melanoma patients, we generated and validated an algorithm based on machine 
learning that could assist with the clinical decision of whether or not to apply ICI therapy by defining five signatures of predictability with $95 \%$ accuracy.

Keywords: ipilimumab; nivolumab; pembrolizumab; melanoma; checkpoint inhibitors; BRAF/MEK inhibitors; survival random forest model

\section{Introduction}

In the past 10 years, we have seen the evolution of melanoma treatment attributed to the development of novel immunotherapy agents that target specific immune regulatory checkpoints, which have completely changed the perspective for metastatic melanoma patients by increasing survival rates and improving quality of life [1-6]. In this context, two immune inhibitory molecules involved in immunosuppressive response have been actively studied: cytotoxic T-lymphocyte-associated antigen-4 (CTLA-4), which helps to extinguish the immune activator signal, and programmed cell death protein 1 (PD-1), which negatively regulates T-cell activation and inhibition of effector function [7]. Based on evidence from prior studies, the idea was born that specific antibodies suppressing inhibition of the immune system in the cancer microenvironment could prevent the inactivation of an effector antitumor immune response [8]. Ipilimumab-a monoclonal antibody (IgG1) directed against CTLA-4 - was the first immunotherapy for metastatic melanoma to have shown a benefit to overall survival (OS) in approximately $20 \%$ of patients in a randomized phase III trial [9]. Nivolumab and pembrolizumab - monoclonal antibodies directed against PD-1-are other immunomodulating agents able to reactivate innate antitumor immunity, eliciting objective responses in a substantial percentage of patients with melanoma [10-13]. However, only a portion of patients will benefit from immunotherapies and, although many studies have been carried out to identify potential predictive and/or prognostic biomarkers useful to identify patients who respond to therapies [14-16], no universally recognized biomarkers are available to date. There is a lack of prognostic biomarkers, and this is one of the main limitations affecting the use of these immunomodulating antibodies. There are described prognostic clinical variables, but there are no comprehensive ways to keep them under a common denominator related to the benefit of interactive treatment. Some examples have been previously described [17-19]. In addition, most efficacy data related to the use of immunomodulating antibodies are derived from randomized trials; their real-life application might give different outcomes compared to the results from clinical trials, as the inclusion and exclusion criteria might be selective and give overoptimistic survival rates. Here, we present real-world data related to 578 metastatic melanoma patients treated at the INT-NA with the immune checkpoint inhibitors (ICIs) ipilimumab, nivolumab, or pembrolizumab as monotherapies to investigate whether patients' baseline clinical characteristics could predict their response to treatment.

\section{Materials and Methods}

\subsection{Study Population}

We retrospectively investigated, from 2012 to 2018, 578 stage IV cutaneous (excluded mucosal and ocular) melanoma patients treated with anti-CTLA-4 (ipilimumab) or anti-PD1 (pembrolizumab or nivolumab) as monotherapy at the INT-NA (Figure 1) (Table 1) [20]. Ipilimumab was administered intravenously at a dosage of $3 \mathrm{mg} / \mathrm{kg}$ every 3 weeks for 4 doses, pembrolizumab at a dosage of $200 \mathrm{mg}$ every 3 weeks, and nivolumab at a dosage of $3 \mathrm{mg} / \mathrm{kg}$ every 2 weeks, until disease progression or unacceptable toxicity appeared. Disease evaluation was performed at baseline and, subsequently, every 12 weeks until progression or the discontinuation of treatment according to the Response Evaluation Criteria in Solid Tumors (RECIST 1.1) [21]. Based on the availability of the data reported in clinical records, clinical variables such as sex, age, BRAF status, LDH, NLR, CNS (central nervous system) metastases, and eosinophils used in routine for metastatic stage 
IV melanoma patients are presented in Table $1 . \mathrm{LDH}$ values were grouped according to the local laboratory reference (LLR) interval: normal $=1 \times$ LLR; high $>1 \times<2 \times$ LLR; very high $>2 \times$ LLR. The NLR was calculated by dividing the absolute counts of neutrophils by the absolute counts of lymphocytes. The range was considered normal with a ratio between 1 and 4 , low $<1$, and high $>4$.

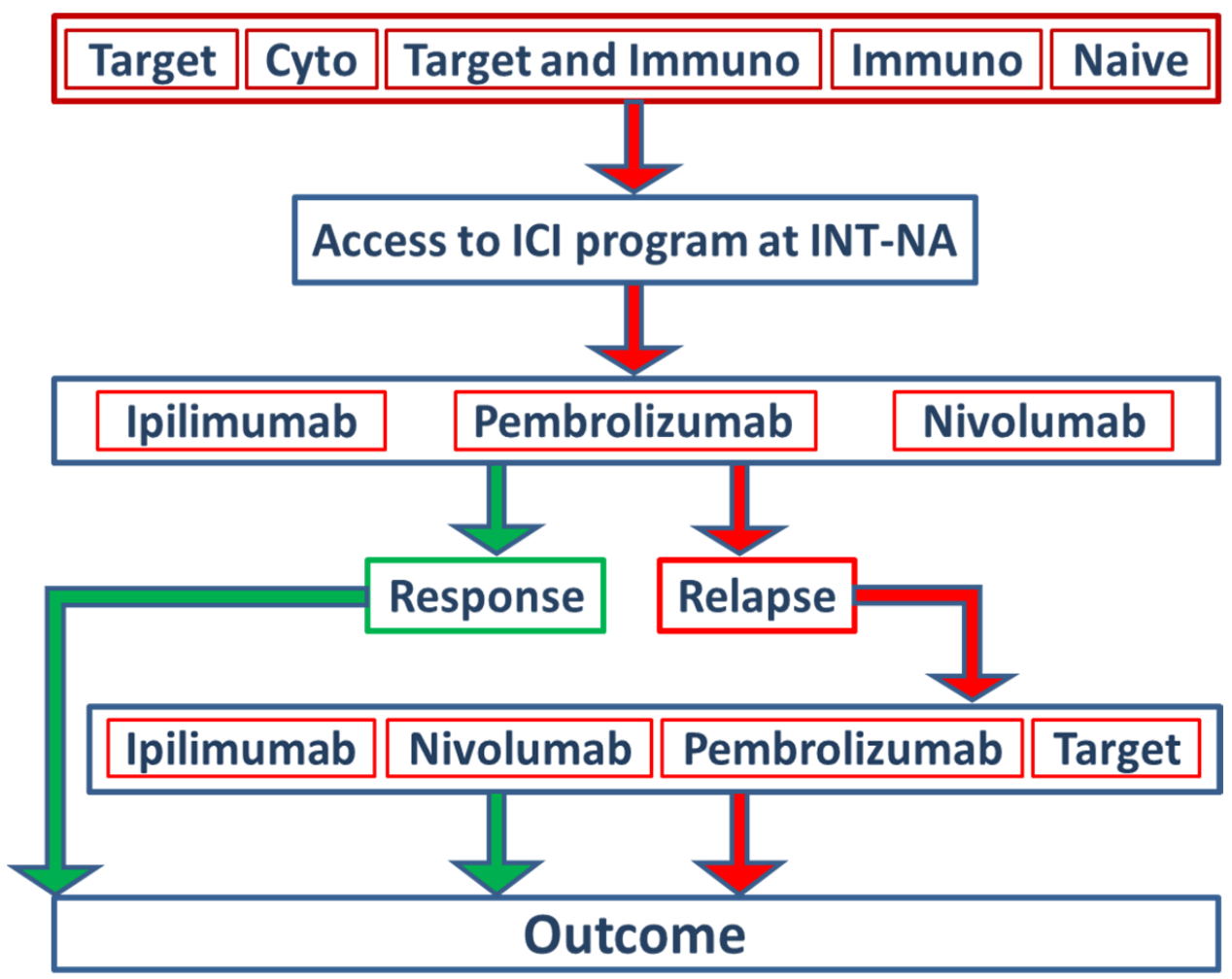

Figure 1. Study design and stratification of patients within the ICI program at INT-NA. In total, 578 stage IV melanoma patients treated with ipilimumab (51\%), pembrolizumab (23\%) or nivolumab $(26 \%)$ as monotherapies at the INT-NA were included in the present study. Based on the type of treatment received before inclusion in the ICI program at the INT-NA, patients were stratified into five groups: target, cytostatic, target and immunotherapy, immunotherapy, and naïve.

Anti-CTLA-4 (ipilimumab 51\%) and anti-PD-1 (nivolumab 26\%; pembrolizumab 23\%) were defined as intervention variables for the ICI program. The distribution between females and males was equally balanced. Furthermore, patients were stratified into five groups based on the type of treatment they had received before they were included in the ICI program at the INT-NA (Figure 1, Table 1). A total of $34 \%$ percent of patients did not receive any therapy before inclusion (naïve), $25 \%$ received prior ICI treatments, $18 \%$ received prior target therapy (TT), $10 \%$ of patients received an ICI and TT, and $13 \%$ of patients were treated with cytostatic schedules. For further predictive and prognostic analyses, the types of pretreatment agents were dichotomized in no target and target subgroups. 
Table 1. Clinical-pathological characteristics of cutaneous melanoma patients. Description of the patient population and inclusion criteria adapted to the real-life situation.

\begin{tabular}{|c|c|c|c|c|c|c|}
\hline \multirow[t]{2}{*}{ Variables } & \multicolumn{2}{|c|}{ All } & \multicolumn{2}{|c|}{ Females } & \multicolumn{2}{|c|}{ Males } \\
\hline & $n$ & $\%$ & $n$ & $\%$ & $n$ & $\%$ \\
\hline \multicolumn{7}{|l|}{ Clinical anamnestic variable } \\
\hline Sex & 578 & 100 & 258 & 45 & 320 & 55 \\
\hline Median age (range) & \multicolumn{2}{|c|}{$61.4(23-89)$} & \multicolumn{2}{|c|}{$59.9(23-89)$} & \multicolumn{2}{|c|}{$62.5(22-87)$} \\
\hline Age 65-100 & 317 & 55 & 132 & 51 & 185 & 58 \\
\hline Age 18-64 & 261 & 45 & 126 & 49 & 135 & 42 \\
\hline BRAF mut $600 \mathrm{E}$ tested & $548^{a}$ & 100 & 241 & 100 & 307 & 100 \\
\hline BRAF 600E mutated & 234 & 43 & 108 & 45 & 126 & 41 \\
\hline CNS met & 572 & 100 & 257 & 100 & 315 & 100 \\
\hline Yes & 162 & 28 & 71 & 28 & 91 & 29 \\
\hline No & 410 & 72 & 186 & 72 & 224 & 71 \\
\hline $\mathrm{LDH}$ & 535 & 100 & 241 & 100 & 294 & 100 \\
\hline Very high $(>2 \times$ LLR) & 77 & 14 & 39 & 16 & 38 & 13 \\
\hline $\operatorname{High}(\geq 1 \times \leq 2 \times$ LLR $)$ & 107 & 20 & 43 & 18 & 64 & 22 \\
\hline Normal $(<1 \times$ LLR) & 351 & 66 & 159 & 66 & 192 & 65 \\
\hline Eosinophil counts & 543 & 100 & 242 & 100 & 301 & 100 \\
\hline Elevated & 47 & 9 & 18 & 7 & 29 & 10 \\
\hline Normal & 496 & 91 & 224 & 93 & 272 & 90 \\
\hline NLR & 551 & 100 & 248 & 100 & 303 & 100 \\
\hline Abnormal & 247 & 45 & 104 & 42 & 143 & 47 \\
\hline Normal & 304 & 55 & 144 & 58 & 160 & 53 \\
\hline \multicolumn{7}{|l|}{ Treatment groups } \\
\hline None (Naïve) & 199 & 34 & 83 & 32 & 116 & 36 \\
\hline Immunotherapy & 142 & 25 & 61 & 24 & 81 & 25 \\
\hline Target and immunotherapy & 59 & 10 & 29 & 11 & 30 & 9 \\
\hline Target & 102 & 18 & 48 & 19 & 54 & 18 \\
\hline Cytostatic & 76 & 13 & 37 & 14 & 39 & 12 \\
\hline \multicolumn{7}{|l|}{$\begin{array}{l}\text { Grouped in Pre-target } \\
\text { /No target }\end{array}$} \\
\hline No target & 417 & 72 & 181 & 70 & 236 & 74 \\
\hline Pre-target & 161 & 28 & 77 & 30 & 84 & 26 \\
\hline \multicolumn{7}{|l|}{$\begin{array}{l}\text { Treatment at inclusion to } \\
\text { the INT-NA ICI program }\end{array}$} \\
\hline Anti-CTLA-4 & 292 & 51 & 129 & 50 & 163 & 51 \\
\hline Anti-PD-1 (nivolumab) & 151 & 26 & 68 & 26 & 83 & 26 \\
\hline Anti-PD-1 (pembrolizumab) & 135 & 23 & 61 & 24 & 74 & 23 \\
\hline
\end{tabular}

a 30 patients lack test results for BRAF 600E mutation: naïve (9), cytostatic (22), or immunotherapy (5); hence, none of them received target therapy before inclusion. 


\subsection{Statistics}

\subsubsection{Survival Analysis}

The $\chi 2$ trend test was used to examine patient characteristics for discrete categorical variables or factors. Three time-related statistical events have been considered in this study with regard to overall survival, with a statistical event defined as death from any cause; survival time was calculated using date of treatment start and date last seen or date of death (end of follow-up). Cumulative survival plots and time-to-event curves were constructed using the Kaplan-Meier product limit method, with the log-rank test applied to detect differences between groups. Univariate Cox regression analyses were performed for each prognostic factor. Hazard ratios (HRs) and 95\% confidence intervals (CIs) were estimated. To test the assumption of proportional hazards, an interaction term of a prognostic variable and a time-dependent covariate were added. A significant effect of that interaction term denotes the presence of a time-dependent effect and, thus, a violation of the proportional hazards assumption. Multivariate Cox regression analyses were performed including binary coding of all factors with a stepwise procedure. $p$-values $<0.05$ were considered statistically significant. All analyses were performed with the programs StatView ${ }^{\mathrm{TM}}$ for Windows and SAS Institute Inc. Version 5.0.1.

\subsubsection{The Clinical Categorization Algorithm (CLICAL)}

In the first step of the aggregation of the clinical variables, based on the risk power defined by the Mantel-Cox analysis, a simple algorithmic score was created. Depending on the dichotomous or trichotomous category and the result of risk value found in a multivariate test, a weight that defines a favorable ( 3 or 2 ; low) or unfavorable ( 1 ; high) risk, was given to each variable chosen to build the algorithm. The final value, named predictive score (CLICAL SCORE), was calculated by summing the weight of each variable and dividing it by the number of the variables selected:

$$
\text { CLICAL SCORE }=\sum_{i}^{n} a x_{i}+b x_{i}+\ldots n x_{i} / n
$$

where $n=$ the number of variables; $a x n x=$ the specific variables; and $i=$ the weight given: 1,2 , or 3 .

In order to let the algorithm reach a high performance of prediction, all variables for each patient are expected to be given (no missing info). Based on this, 503 out of 578 patients were considered. The scores were grouped into predictive signatures (CLICAL SIGNATURE) from the worst benefit (Signature I) to the best benefit (Signature V). The variables and their relative weights were age (younger vs. older-1 and 2, respectively), BRAF (mutation vs. wild type-1 and 2, respectively), pretreatment with TT (yes vs. no-1 and 2, respectively), LDH (very high vs. high vs. normal-1, 2, and 3, respectively), NLR (abnormal vs. normal-1 and 2, respectively), and eosinophil percentage (abnormal vs. normal - 1 and 2, respectively). The CLICAL methodology was applied to an external cohort of 117 patients (103 out 117 were naïve at inclusion) recruited with the same inclusion criteria at the Department of Oncology, Karolinska University Hospital, Sweden [22]. The CLICAL could significantly separate signatures of prediction for different groups of patients with the same efficiency observed in the INT-NA cohort.

\subsubsection{Application of the Machine Learning Survival Random Forest Clinical} Categorization Algorithm (SRF-CLICAL) and Definition of Prognostic Signatures

The original CLICAL was further analyzed for the development and validation of a "proof-of-concept" algorithm using artificial intelligence (AI) methods, in particular using machine learning through the implementation of the survival random forest (SRF) model [23]. 


\subsubsection{Cox Proportional Hazards Analysis}

Univariate Cox proportional hazards (Cox PH) regression models were fitted for all eight variables listed in Table 1, with survival time being used as the outcome variable, using the R package survival v. 3.2-3 [24]. Effron approximation was used for handling tied death times. The $p$-values and hazard ratios of the models were inspected to compare the predictive abilities of the independent variables. Multivariate Cox $\mathrm{PH}$ models were then fitted using all eight variables. Forest plots were generated to visualize the results using the ggforest function (forest plot for Cox proportional hazards model) of the $\mathrm{R}$ package survminer v. 0.4.8 [25]. Cox PH model performance was assessed for seven clinical variables, after excluding sex as an insignificant variable, by dividing the dataset into training and validation sets (comprised of $80 \%$ and $20 \%$ of the cohort, respectively). The $R$ package pec v. 2019.11.03 [26] was then used for making predictions for the validation set based on the Cox PH model, and for calculating prediction errors and C-indices. The riskRegression package v. 2020.02.05 [27] was used for plotting time-dependent ROC curves and calculating AUC values.

\subsubsection{Survival Random Forest Model}

The SRF model was computed for the data using the following seven variables as features: age group ( $\leq 60$ or $>60$ years), BRAF mutation status, LDH levels, presence of CNS metastasis, previous treatment type, eosinophil levels, and NLR (see also Table 1). The R package randomForestSRC v. 2.9.3 [28] was used for computing the model using the training dataset ( $80 \%$ of the cohort). An optimized SRF model was generated by tuning mtry and node size parameters for 50,100, 200, 500, and 1000 trees using the tune.rfsrc function of the randomForestSRC package, with the starting value of mtry set to 2 . Out-ofbag (OOB) errors of the models were compared, and the number of trees with the smallest OOB error (ntree $=1000$ ) was chosen as the ntree value for the optimized SRF model, with optimal mtry $=2$ and nodesize $=10$ values for the given number of trees used for generating the final model. The R package pec v. 2019.11.03 [26] function predictSurvProb was then used for making survival probability predictions for the $20 \%$ validation set at 12, 24, 36, and 60 months. The riskRegression package v. 2020.02.05 [27] was used for plotting time-dependent ROC curves as in the previous assessment of the Cox PH model's performance. Similarly, an SRF model was also computed using the full dataset. The parameters of the optimized SRF model for the full dataset were ntree $=500$, try $=2$, and node size $=6$.

\subsubsection{Kaplan-Meier Survival Curves}

Kaplan-Meier plots were generated using the R packages survival v. 3.2-3 [24] and survminer v. 0.4.8 [25] for patients divided into three risk groups based on the SRFpredicted survival probabilities. For that, the full dataset was used to generate an optimized SRF model and make predictions of survival probability for each patient. The full dataset was used for this analysis so that an adequate number of patients could be assigned to each group. Distribution of the predicted survival probabilities at 5 years (60 months) was examined and used to define the risk group categories of the patients: patients with survival probability $<0.2$ were categorized into the high-risk group, patients with survival probability $\geq 0.41$ were categorized into the low-risk group, and patients with survival probability in between these thresholds were categorized into the medium-risk group. The patients were further stratified according to their treatment group (anti-CTLA-4 or anti-PD-1).

\section{Results}

\subsection{Clinicopathological Features of Melanoma Patients}

A total of 578 stage IV cutaneous melanoma patients ( 323 males, 255 females, median age 61.2) were included in the present study (Figure 1) (Table 1); 292 out of 578 patients (51\%) received ipilimumab as monotherapy, 151 out of 578 patients (26\%) received nivolumab as 
monotherapy, and 125 out of 578 patients (23\%) received pembrolizumab as monotherapy. The clinical variables of the patients are presented in Table 1 . The distribution is also specified for females and males. Additionally, the age had a cutoff at 65 years separating the group into younger and older, with a slightly higher representation among the patients aged 65 and over. In the male group, $58 \%$ were older patients compared to $42 \%$ of younger males, and the difference was not significant. For 548 patients $(94.8 \%)$, the presence of BRAF mutation at the codon $600 \mathrm{E}$ was analyzed, while 30 patients were not tested for BRAF mutation. A total of $43 \%$ of patients had a detectable mutation in BRAF, with no statistical significance between sexes. CNS metastases were present in $28 \%$ of the cases included in the analysis, with an equal distribution between sexes. LDH values were grouped according to the local laboratory reference (LLR) interval: normal $=1 \times$ LLR; high $>1 \times<2 \times$ LLR; very high $>2 \times$ LLR. The level of LDH was detected as very high in $14 \%$ of the cases, high in $20 \%$ of patients, and normal in $66 \%$ of the patients. Only $9 \%$ of patients had elevated eosinophils in their circulating blood. The NLR was abnormal in $45 \%$ of the patients. No difference between females and males was registered for these peripheral blood parameters.

\subsection{The Efficacy of ICI Depending on the Previous Treatment}

The analysis of the OS of the population of patients studied is presented in Figure 2. Taken together (for any type of intervention ICI), the entire cohort of 578 cases had an OS of $20 \%$ at 70 months (Figure 2a). Applying the different categories of treatment (as defined in Table 1), naïve and immunotherapy pretreated patients had the highest OS (Figure 2b). The patient groups that received TT only or ICI and TT before the start of the study had the worst outcomes. OS analysis of patients included in the program at the INT-NA, categorized based on ICI treatment (anti-CTLA-4 and anti-PD-1), is shown in Figure 3. As expected, the anti PD-1 strategy showed a better impact in the final outcome compared to anti-CTLA-4 treatment (Figure $3 a, p=0.002$ ), and it was particularly efficacious in naïve patients (Figure $3 b, p=0.0002$ ).
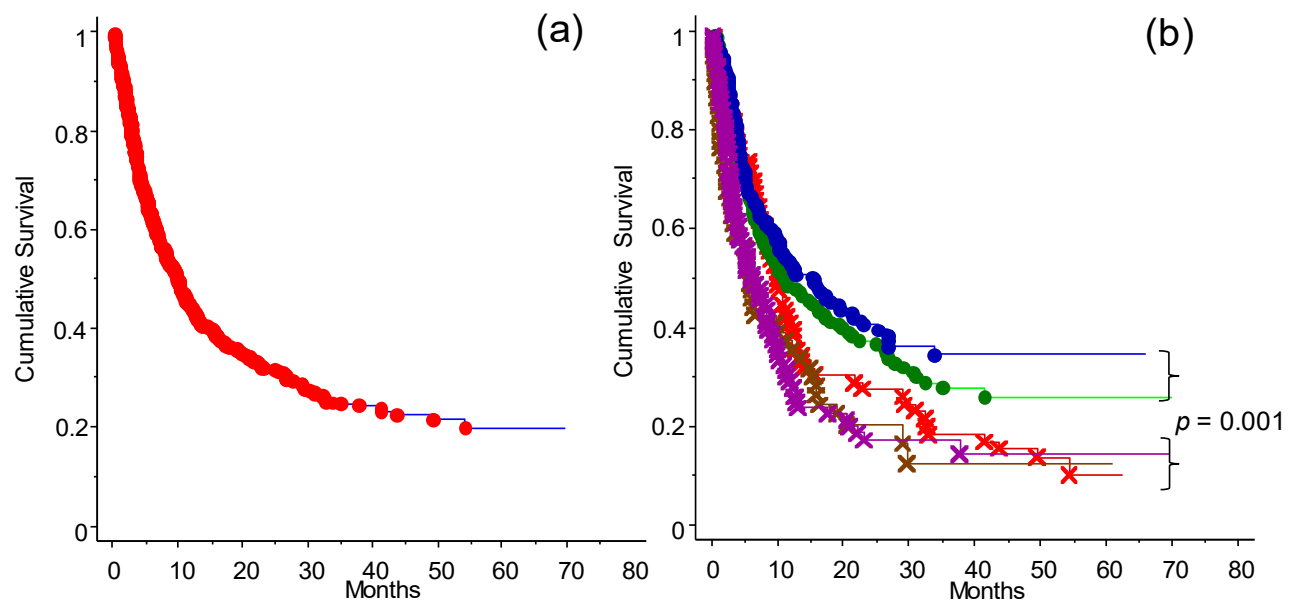

Figure 2. Cumulative survival analysis of patients depending on treatment before enrollment to the ICI program at the INT-NA: (a) Survival curve for the whole cohort of patients $(n=578)$. (b) Survival analysis of patients stratified into five groups based on treatment received before access to the INTNA's program: naïve (blue line); immunotherapy (green line), cytostatic (red line), immunotherapy and target treatment (brown line), and target treatment (purple line). Naïve and immunotherapy groups (non-target) had an OS significant higher compared to the other treatment groups $(p=0.001)$ (see also Figure $4 \mathrm{a}$ ). 

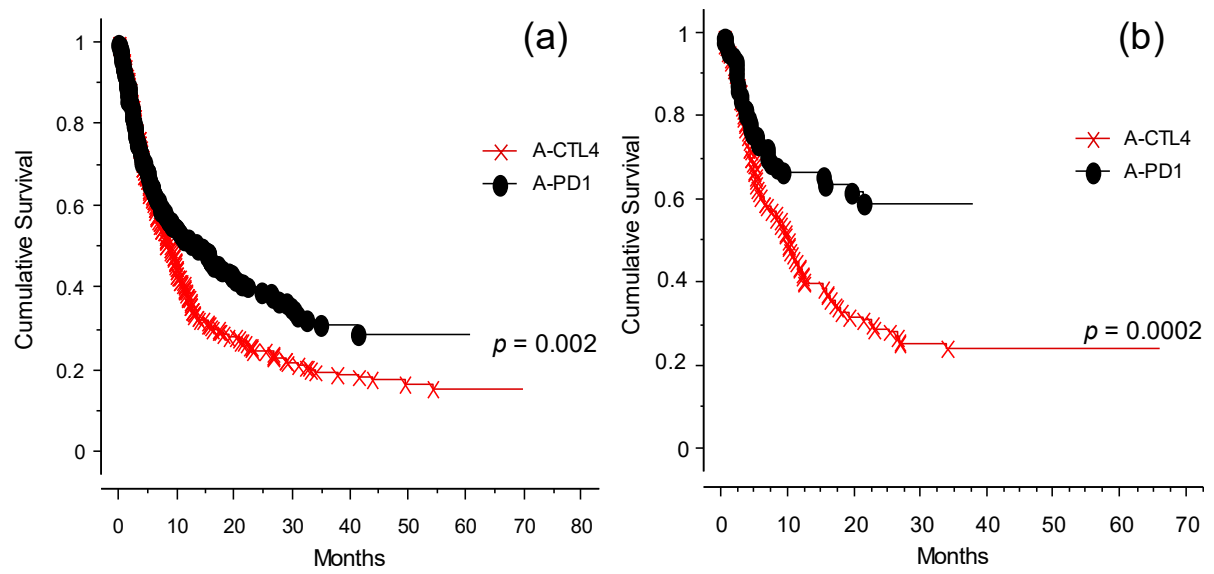

Figure 3. OS analysis for anti-CTLA-4- or anti-PD-1-treated patients (observation time $>40$ months): (a) OS analysis for the whole cohort of patients categorized by type of ICI received at INT-NA: anti-CTLA-4 $(\times-\times)$ or anti-PD-1 $(\bullet-\bullet), p=0.002$. (b) OS analysis of the naïve group selected for type of ICI received at INT-NA: anti-CTLA-4 $(\times-\times)$ or anti-PD-1 $(\bullet-\bullet), p=0.0002$.

\subsection{The Response to Immunotherapy}

The analysis of response to the ICIs is presented in Tables 2 and 3. Table 2 shows the analysis of relapse-free survival (RFS) and OS for all patients, while Table 3 shows only naïve patients.

Table 2. RFS and OS analysis of the whole cohort. Probability of relapse free survival and probability of survival after immunotherapy received at inclusion at the INT-NA.

\begin{tabular}{|c|c|c|c|c|c|c|c|c|c|}
\hline & \multirow[b]{2}{*}{$n(\%)$} & \multicolumn{4}{|c|}{ RFS } & \multicolumn{4}{|c|}{ OS } \\
\hline & & $\begin{array}{l}\text { Probability } \\
\text { of RFS (\%) at } \\
24 \text { Months }\end{array}$ & $\begin{array}{c}\text { CI } 95 \% \\
\text { Min-Max }\end{array}$ & $\begin{array}{l}\text { Cumulative } \\
\text { Hazard at } \\
24 \text { Months }\end{array}$ & $\begin{array}{c}\text { CI } 95 \% \\
\text { Min-Max }\end{array}$ & $\begin{array}{c}\text { Probability } \\
\text { of Survival } \\
\text { (\%) at } \\
50 \text { Months }\end{array}$ & $\begin{array}{c}\text { CI } 95 \% \\
\text { Min-Max }\end{array}$ & $\begin{array}{l}\text { Cumulative } \\
\text { Hazard at } \\
50 \text { Months }\end{array}$ & $\begin{array}{c}\text { CI 95\% } \\
\text { Min-Max }\end{array}$ \\
\hline ALL & $578(100)$ & 16 & $13-19$ & 1.83 & $1.63-2.03$ & 8 & 5-11 & 2.46 & 2.12 .80 \\
\hline CR & $32(5.5)$ & 67 & $48-85$ & 0.39 & $0.12-0.66$ & 81 & $61-100$ & 0.19 & $0-0.43$ \\
\hline PR & $74(12.8)$ & 64 & $52-76$ & 0.48 & $0.28-0.67$ & 58 & $42-75$ & 0.52 & $0.24-0.79$ \\
\hline SD & 103 (17.8) & 24 & $15-33$ & 1.48 & $1.09-1.86$ & 39 & $25-52$ & 0.94 & $0.60-1.28$ \\
\hline PD & $369(63.8)$ & 0 & 0 & 6.42 & $3.91-8.93$ & 5 & $2-7$ & 2.9 & $2.4-3.4$ \\
\hline
\end{tabular}

Table 3. RFS and OS analysis of naïve cohort. Probability of relapse free survival and probability of survival after immunotherapy received at inclusion at the INT-NA.

\begin{tabular}{|c|c|c|c|c|c|c|c|c|c|}
\hline & \multirow[b]{2}{*}{$n(\%)$} & \multicolumn{4}{|c|}{ RFS } & \multicolumn{4}{|c|}{ OS } \\
\hline & & $\begin{array}{l}\text { Probability } \\
\text { of RFS (\%) at } \\
24 \text { Months }\end{array}$ & $\begin{array}{c}\text { CI 95\% } \\
\text { Min-Max }\end{array}$ & $\begin{array}{l}\text { Cumulative } \\
\text { Hazard at } \\
20 \text { Months }\end{array}$ & $\begin{array}{c}\text { CI 95\% } \\
\text { Min-Max }\end{array}$ & $\begin{array}{c}\text { Probability } \\
\text { of Survival } \\
\text { (\%) at } \\
50 \text { Months }\end{array}$ & $\begin{array}{c}\text { CI 95\% } \\
\text { Min-Max }\end{array}$ & $\begin{array}{l}\text { Cumulative } \\
\text { Hazard at } \\
50 \text { Months }\end{array}$ & $\begin{array}{c}\text { CI 95\% } \\
\text { Min-Max }\end{array}$ \\
\hline ALL & $199(100)$ & 22 & $16-28$ & 1.49 & $1.22-1.76$ & 36 & $27-42$ & 1.06 & $0.83-1.28$ \\
\hline CR & $17(8.5)$ & 81 & $62-100$ & 0.32 & $0-0.66$ & 100 & 100 & 0 & 0 \\
\hline PR & $29(14.5)$ & 73 & $56-90$ & 0.31 & $0.08-0.53$ & 76 & $52-99$ & 0.27 & $0-0.56$ \\
\hline SD & 36 (17.6) & 31 & $14-43$ & 1.13 & $0.61-1.64$ & 55 & $34-76$ & 0.58 & $0.21-0.96$ \\
\hline PD & $118(59.3)$ & 0 & 0 & 4.28 & $2.73-5.83$ & 11 & $6-17$ & 2.16 & $1.61-2.71$ \\
\hline
\end{tabular}




\subsection{Analysis of the Factors Related to the Efficacy of Immunotherapy (ICI Program)}

Role of the Different Treatments Given before Inclusion to the INT-NA

In Figure 4 the role of the treatments received by patients before treatment with ICI at the INT-NA is shown, with particular regard to TT. The previous treatments have been grouped in target or non-target (Figure 4a). The group of patients that received TT responded poorly $(p<0.0001)$. Interestingly, in this group the effect of anti-PD-1 was not significantly different from that of anti-CTLA-4 (Figure $4 b, p=0.07$ ). Looking at antiCTLA-4- and anti-PD-1-treated patients, those who did not receive previous TT had more favorable outcomes in both cases (Figure $4 \mathrm{c}, p=0.002$ ).
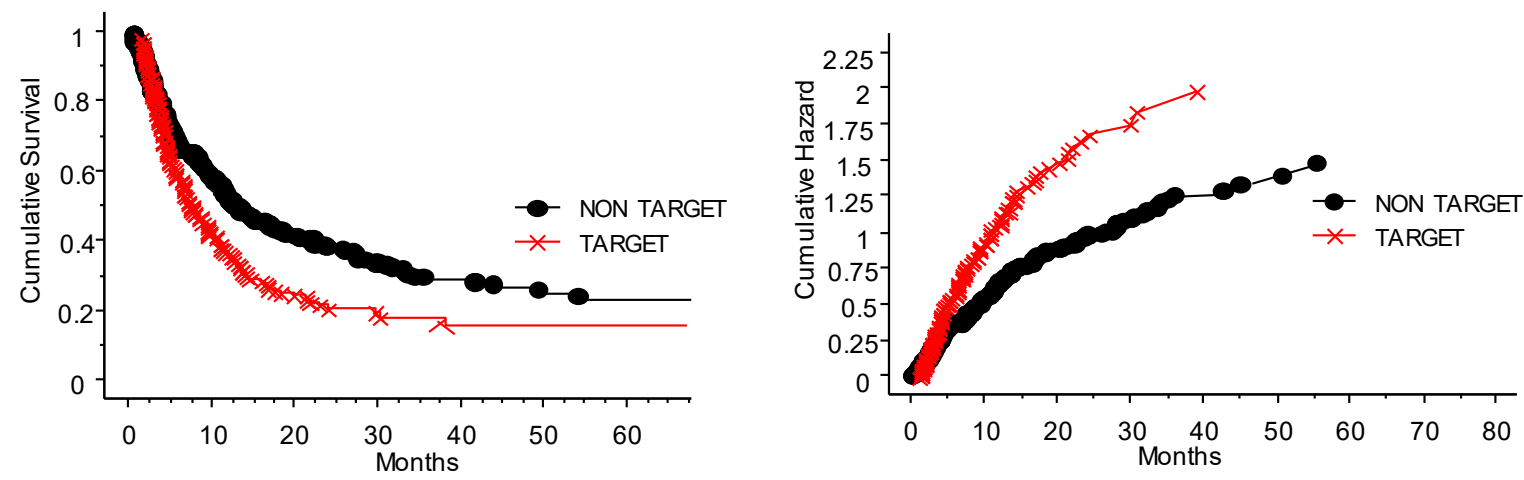

(a)
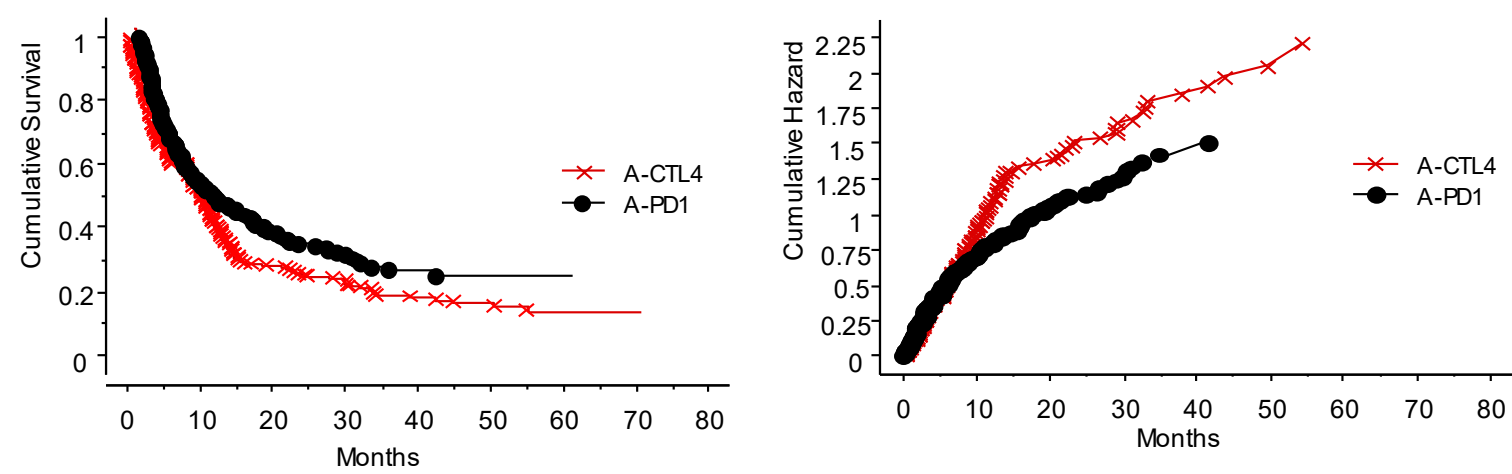

(b)
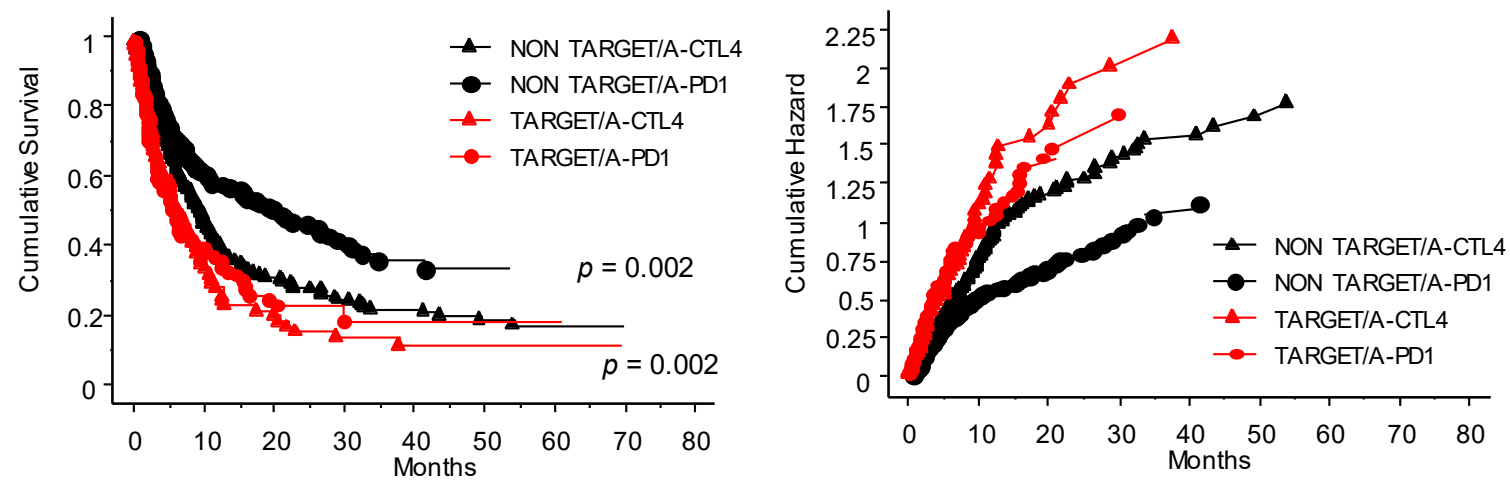

(c)

Figure 4. Interaction and significance of the treatments received before inclusion in the ICI program at the INT-NA: cumulative survival (left panels) and hazard plots (right panels). (a) The treatments before inclusion (see Figure 2b) have been simplified by grouping them into target (red line) or non-target (black line) groups. Patients who did not receive target treatment had more favorable outcomes $(p<0.0001)$. (b) In the cohort of patients who received prior TT, when exposed to anti-CTLA-4 $(\times)$ or anti-PD-1 $(\bullet)$ (i.e., naïve not included), interestingly, the effect of anti-PD-1 was not significantly different from that of anti-CTLA-4 $(p=0.07)$. (c) Significance of first-line treatment with target or non-target therapy on the outcome of the ICI program. In both anti-CTLA-4 $(\Delta)$ and anti-PD1 $(\bullet)$ treatments, patients who did not receive previous target treatment had a more favorable outcome $(p=0.002)$. 


\subsection{Treatment of Patients Who Relapsed after the ICI Program at the INT-NA}

The probability of survival and chance of good response for patients without further treatment was significantly different (Figure $5, p=0.0001$ ) from other intervention strategies (treatments other than ICIs and TT after relapse, ICIs after relapse, TT after relapse, or no further treatment) (Figure 5). It is also important to note that the patients treated with other therapies after disease relapse had a clinical benefit compared to patients who were not treated after relapse. There is no evidence of differences between ICIs or other strategies of treatment after relapse. Thus, when possible, it is advantageous to invest in further treatments.
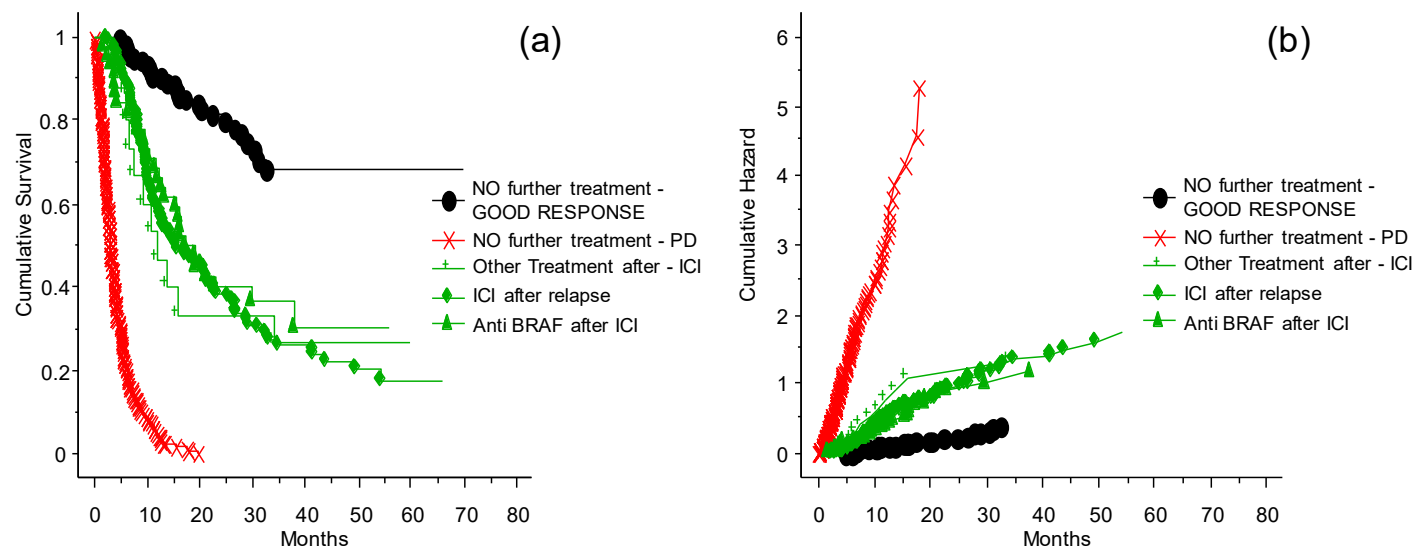

Figure 5. The probability of survival and risk for patients who relapsed after the ICI program at the INT-NA. Panel (a) shows probability of survival, panel (b) shows risk. Subsequent line of treatment or no further treatment, and comparison between TT given after the ICI program.

The results summarized in Figure 6 needs to be scrutinized looking at the presence or absence of BRAF 600E and consequent treatment or not before exposure to ICIs. In fact, patients who were treated with TT prior to ICIs had less opportunity to respond to ICIs, whether it was anti-CTLA-4 or anti-PD-1 therapy (see also Figure 4). The effect of anti-PD-1 was not significantly different from that of anti-CTLA-4. Treatment with anti-PD-1 of patients previously treated with cytostatic drugs or immunotherapy, or naïve patients, produced a better OS. The patients had different benefits depending on whether the treatment with TT was delivered before or after challenge with ICIs $(p<0.0001)$.
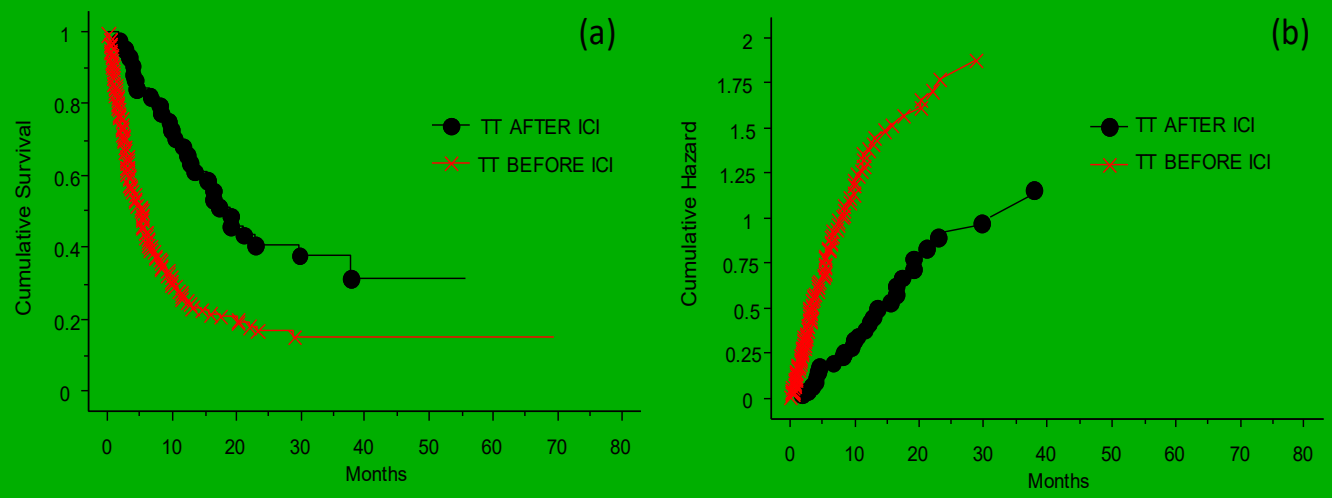

Figure 6. Probability of survival and risk for patients treated with TT before or after ICIs. Panel (a) shows probability of survival, panel (b) shows risk. The patients had different benefits depending on whether the treatment with TT was delivered before (worst OS, red line) or after (better OS, black line) the challenge with ICIs in case of disease progression $(p<0.0001)$. 


\subsection{Analysis of the Predictive Power of Clinical Variables at Inclusion}

The relevant clinical data given by gender, age, BRAF 600E mutation, LDH, CNS metastases, previous TT before the inclusion to ICI program, eosinophil counts, and NLR were initially analyzed for their risk of death due to the metastatic disease via univariate and multivariate Mantel-Cox methods (as explained in the Materials and Methods section). Each of these variables could determine, singularly or in a hierarchical way, the power of prognosis as shown in the forest plot (Figure 7). This preliminary analysis permits us to assign the weight of risk and build the score derived from the CLICAL verified by the SRFCLICAL algorithm (Table 4). Based on hazard ratio, gender was subsequently excluded from the CLICAL. The algorithm calculated eight score levels; these were scrutinized in a survival plot, and those closer to one another were grouped together for the final five signatures (Table 4 and Figure 8a).

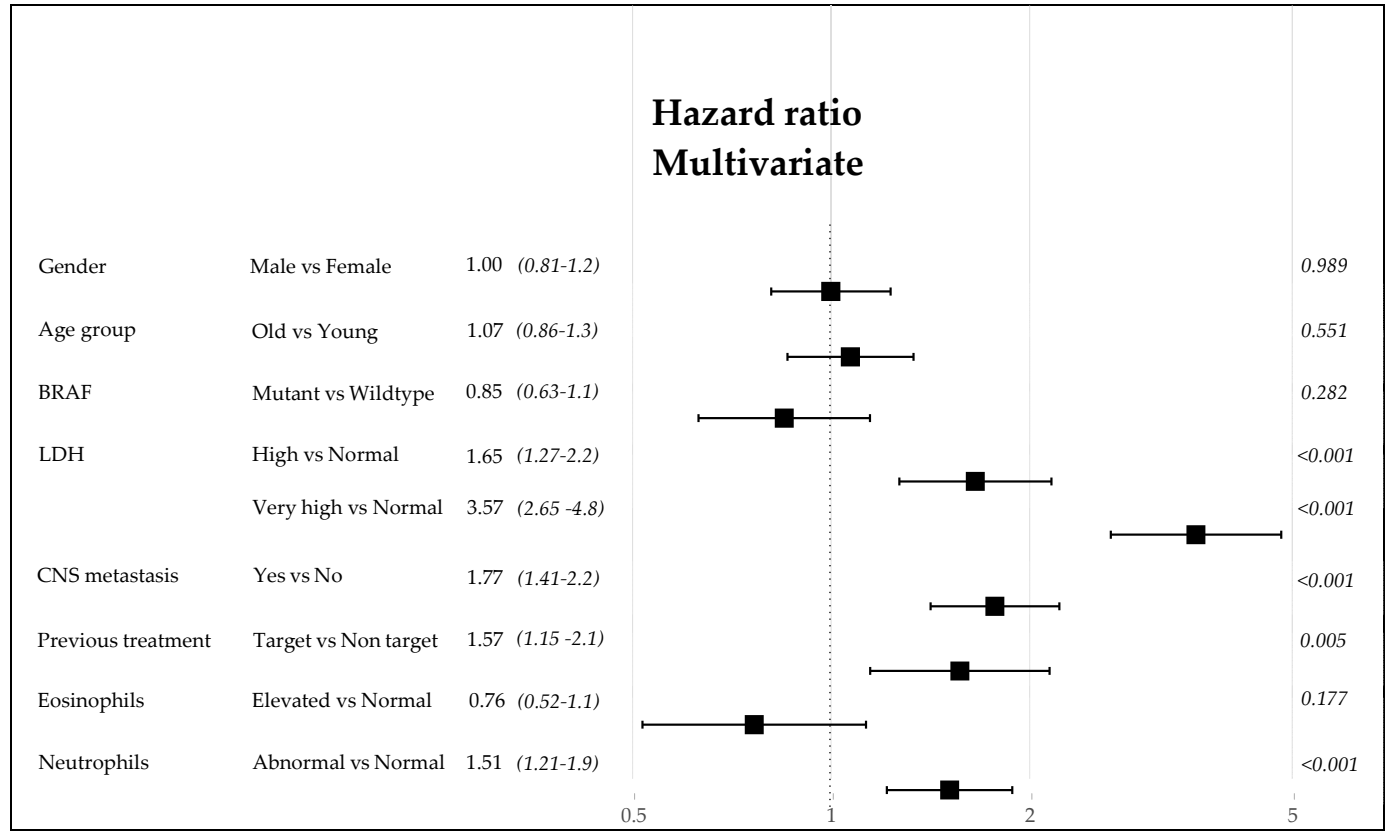

Figure 7. Forest plot of the clinical variables. The hazard ratio and 95\% CI are described as well as the $p$-value.

Table 4. CLICAL algorithm scores and signatures related to 503 out of 578 patients. CLICAL algorithm outcomes in scores and grouped in signatures defined by the cumulative risk at 32 months.

\begin{tabular}{|c|c|c|c|c|c|c|}
\hline $\begin{array}{l}\text { CLICAL } \\
\text { SCORE }\end{array}$ & $n$ & $\begin{array}{c}\text { Cumulative } \\
\text { Hazard at } \\
32 \text { Months } \\
\text { (95\% CI) }\end{array}$ & $\begin{array}{c}\text { Still Alive (at } \\
\text { Risk) at } \\
32 \text { Months }\end{array}$ & $\begin{array}{c}\text { CLICAL } \\
\text { SIGNATURES }\end{array}$ & $n(\%)$ & $\begin{array}{l}\text { Cumulative } \\
\text { Hazard at } \\
32 \text { Months } \\
(95 \% \text { CI })\end{array}$ \\
\hline 1.143 & 8 & $2.7(0.29-5.14)^{\#}$ & 0 & \multirow{2}{*}{ Signature I } & \multirow{2}{*}{$46(9.1)$} & \multirow{2}{*}{$3.42(1.87-4.96)$} \\
\hline 1.286 & 38 & $3.23(1.69-4.77)$ & 1 & & & \\
\hline 1.429 & 71 & $2.41(1.59-3.24)$ & 2 & Signature II & $71(14.1)$ & $2.41(1.59-3.24)$ \\
\hline 1.571 & 111 & $1.70(1.24-2.16)$ & 10 & Signature III & $111(22.1)$ & $1.70(1.24-2.16)$ \\
\hline 1.714 & 127 & $1.14(0.86-1.43)$ & 10 & \multirow{2}{*}{ Signature IV } & \multirow{2}{*}{$243(48.3)$} & \multirow{2}{*}{$1.08(0.89-1.27)$} \\
\hline 1.857 & 116 & $1.00(0.75-1.20)$ & 30 & & & \\
\hline 2.000 & 31 & $0.50(0.19-0.81)$ & 12 & \multirow{2}{*}{ Signature V } & \multirow{2}{*}{$32(6.4)$} & \multirow{2}{*}{$0.48(0.19-0.77)$} \\
\hline 2.143 & 1 & $0.50(0.19-0.81)$ & 1 & & & \\
\hline
\end{tabular}



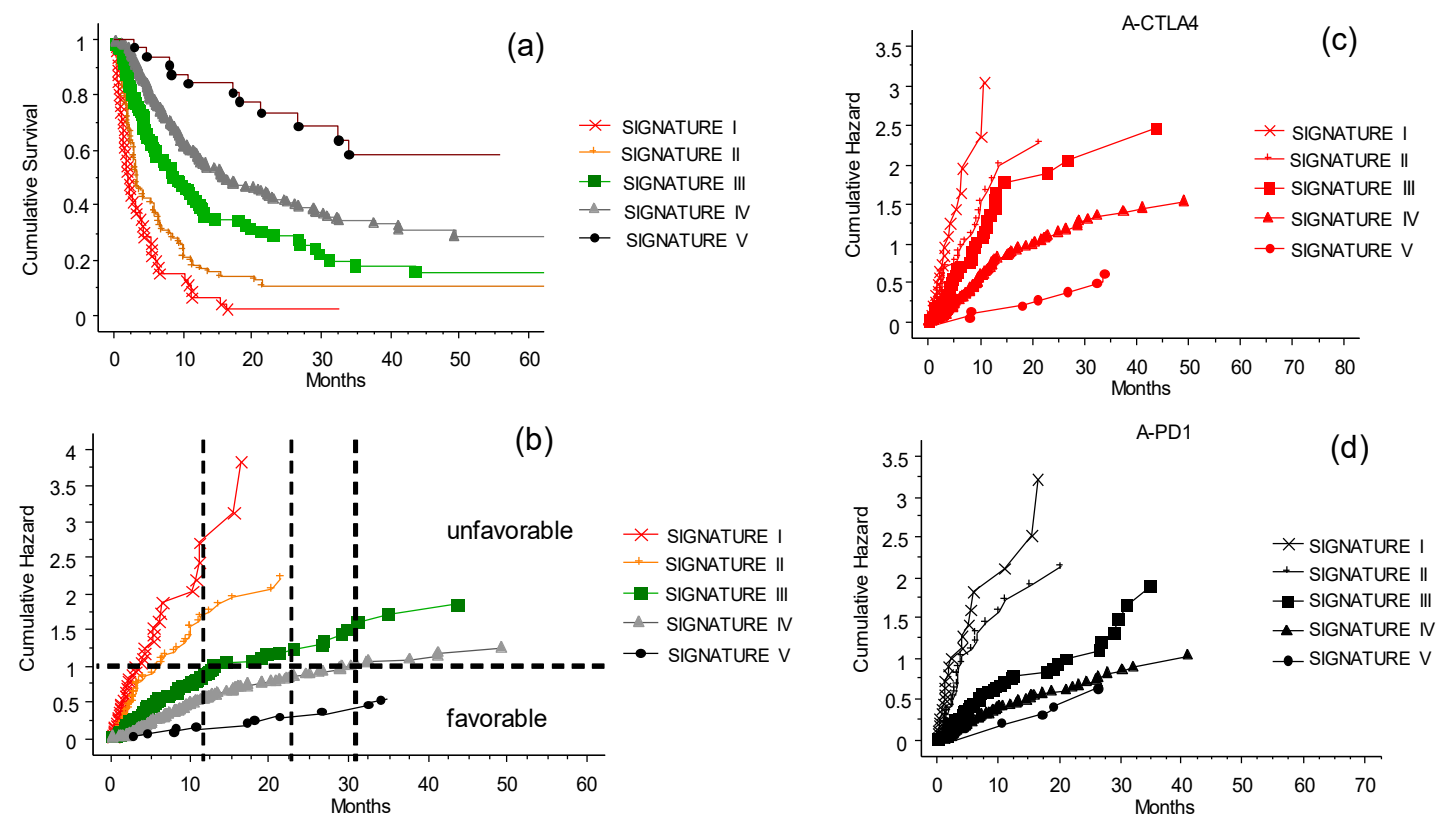

Figure 8. The CLICAL signatures and their interaction with the ICI treatments. Plot (a) shows the cumulative survival with the distribution of the patients grouped by their signatures. The five curves are significantly separated from one another ( $p=0.001$ ). (b) Cumulative risks for each signature easily show whether the characteristics of each signature are favorable or not. Plot (c) presents the effect of the signature when compared with anti-CTLA-4 treatment. The difference in prediction for the five signatures is significant $(p=0.0001)$. (d) Signatures IV and V predict a similar benefit to patients treated with anti-PD-1, and are different from Signature III as well as from I and II ( $p=0.0001)$.

\subsection{The CLICAL Signature and Prediction of Survival Rates}

The CLICAL algorithm has the ability to distinguish groups of patients by their signature. The five signatures had different prediction rates for survival (Figure $8 \mathrm{a}, \mathrm{b}$ ) ( $p=0.001)$; the higher the signature, the better the odds of survival. Signature I, which is built by the highest risk values for each of the variables selected in the algorithm, had no survival after 32 months. On the opposite end, the patients with Signature V, built by the lowest risk values for each variable, had the highest percentage of survival. The plots separated by the different signatures shown in Figure $8 \mathrm{a}, \mathrm{b}$ represent the separation of the survival curve of the whole cohort after the ICI program at the INT-NA, as presented in Figure 2a. The difference in prediction between the signatures is significant $(p=0.0001)$. Looking at anti-CTLA-4 therapy (Figure 8c), the signatures show clearly that patients with lower signatures (I-II) will not benefit from the treatment compared to patients with Signature V. Still, there is a possibility of a longer survival in the group with Signature IV. For comparison, see the prognostic plot for the whole cohort presented above in Figure 3.

The same could be said for the anti-PD1 therapy (Figure 8d), with the difference being that Signature III-V indicates a significant benefit from the therapy. Signatures IV and $\mathrm{V}$ give similar prediction, and significant difference to that of Signature III. Signatures I and II were not associated with a long-lasting benefit. In practice, the use of the signature can provide a tool to decide whether it is beneficial to expose a patient with the lowest signature to both of the intervention ICIs as a second challenge, or instead concentrate on more palliative strategies, avoiding the ICI side effects.

\subsection{The CLICAL Signature and Prediction of Response to ICIs}

The CLICAL algorithm has the ability to distinguish response to ICI treatment of patients by their signature. The five signatures had different prediction rates for response (Figure $9 \mathrm{a}, \mathrm{b})(p=0.001)$. Signature I had lower rates of response and, at the opposite end, the patients with Signature V had the highest percentage of response; the higher the signature, the better the odds of response. The cumulative hazard plot shows that 
patients not responsive to the therapy also have a higher risk to die earlier compared to patients responding to the therapy (Figure 9a). Interestingly, non-responding patients but with higher signatures (Signatures IV and V) have a better chance to survive compared to non-responding patients with lower signatures (Signatures I, II, and III) (Figure 9a). The group of patients with higher signatures (i.e., less and less risk), is composed of an increasing percentage of responsive patients and a decreasing percentage of non-responsive patients compared to the group of patients with lower signatures (Figure $9 b$ ). Of interest is the fact that patients who did not respond to the ICI treatment could still have a chance to live longer if they had a higher signature at inclusion; this could be due to the opportunity to receive subsequent treatment.
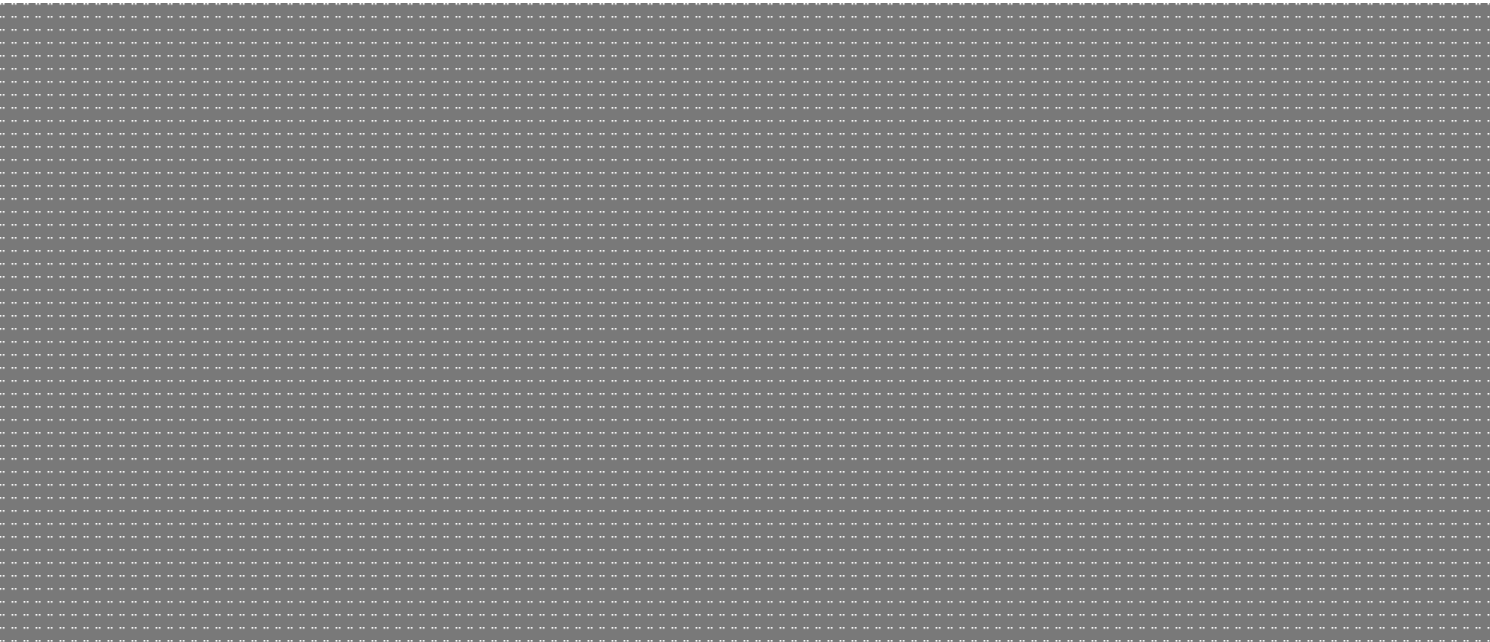

Figure 9. The CLICAL signatures and response to the ICI treatment (complete response (CR), partial response (PR), stable disease (SD); responders), (progressive disease (PD); non-responders). (a) Cross-analysis of the CLICAL signatures panel with the results from ICI intervention. Cumulative risks for each signature easily show whether the characteristics of each signature are favorable or not. (b) Percentage of cases in responders present in each signature after the ICI treatment. Signature V comprises a higher percentage of responder patients and a lower percentage of non-responder patients compared to Signature I. The numbers of cases for each response category and signature are presented in the attached table.

\subsection{The CLICAL Signature Applied to an External Cohort}

The analysis with CLICAL was also applied to an external cohort of 117 patients, available at the Karolinska University Hospital, Sweden. The same categorical variables were studied, and the signatures obtained significantly discriminated the predictive benefits of ICI treatment (Figure 10). In this cohort, the CLICAL could define only four signatures (Signatures I-IV), since the number of available patients with the highest score (only one patient) was not sufficient to build five levels of signature.

\subsection{The Validation of the CLICAL Algorithm's Efficiency by Machine Learning Survival Random Forest Analysis (SRF-CLICAL)}

Prediction performance of the Cox model with seven variables (gender was excluded from the model) was studied using training-validation settings, and time-dependent receiver operating characteristic (ROC) curves at time points 12, 24, 36, and 60 months were generated (Figure 11). The resulting areas under the ROC curves (AUCs) were computed, and are also shown in the plots. As shown in the plots (Figure 11), the AUCs of the sevenvariable Cox models were 71.5, 73.5, and 80.3 at timepoints 1, 2, and 3 years, respectively. At the timepoint of 5 years, the number of cases was so low that a proper, informative ROC curve could not be computed. 
Karolinska Cohort
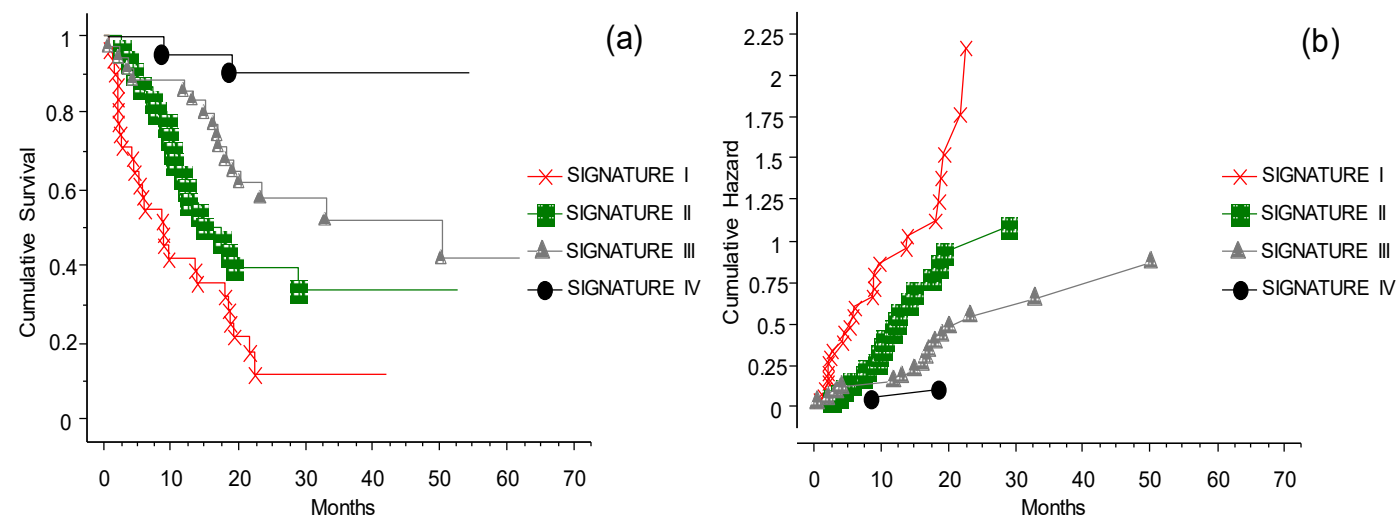

Figure 10. The CLICAL signatures obtained from the external cohort from Karolinska. Due to the restricted numbers, the cases could only be grouped in Signatures I-IV. (a) The plot shows the cumulative survival, with the distribution of the patients grouped by their signatures. The four curves are significantly separated from one another $(p=0.001)$. (b) The presentation of the cumulative risks for each signature easily shows that the CLICAL can determine the characteristics of each signature.

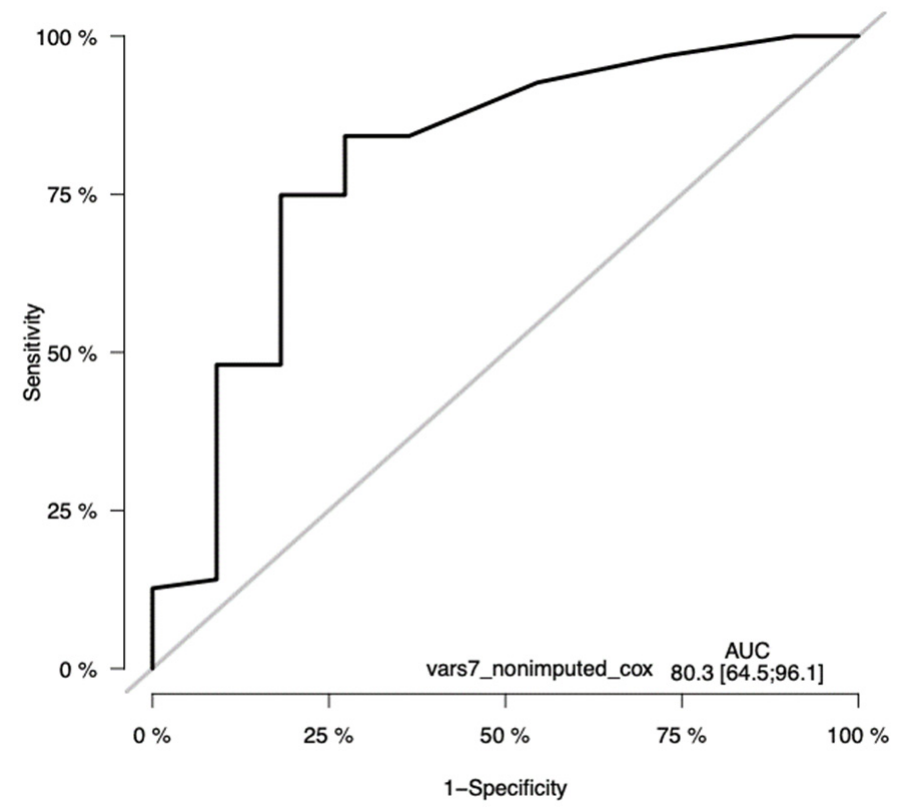

Figure 11. Time-dependent receiver operating characteristic (ROC) curves of the seven-variable Cox $\mathrm{PH}$ model at the timepoint of 36 months. The ROC has a significant sensitivity, with an AUC of 80.23(CI 64.5-96.1). The y-axis in the plot displays the true positive rate (TPR) - i.e., sensitivitywhereas the $x$-axis in the plot displays the false positive rate (FPR), i.e., specificity.

\subsection{Survival Random Forest Model}

Survival random forest (SRF) models were created for predicting patient survival using the same seven clinical variables as the selected features for the models that were used for the Cox model. Optimized SRF models were generated by tuning model parameters and by using similar training - validation settings as for the Cox model, as well as using the full dataset. Due to the available sample size, the ROC curves were generated using the full dataset to obtain an adequate number of patients (Figure 12). 


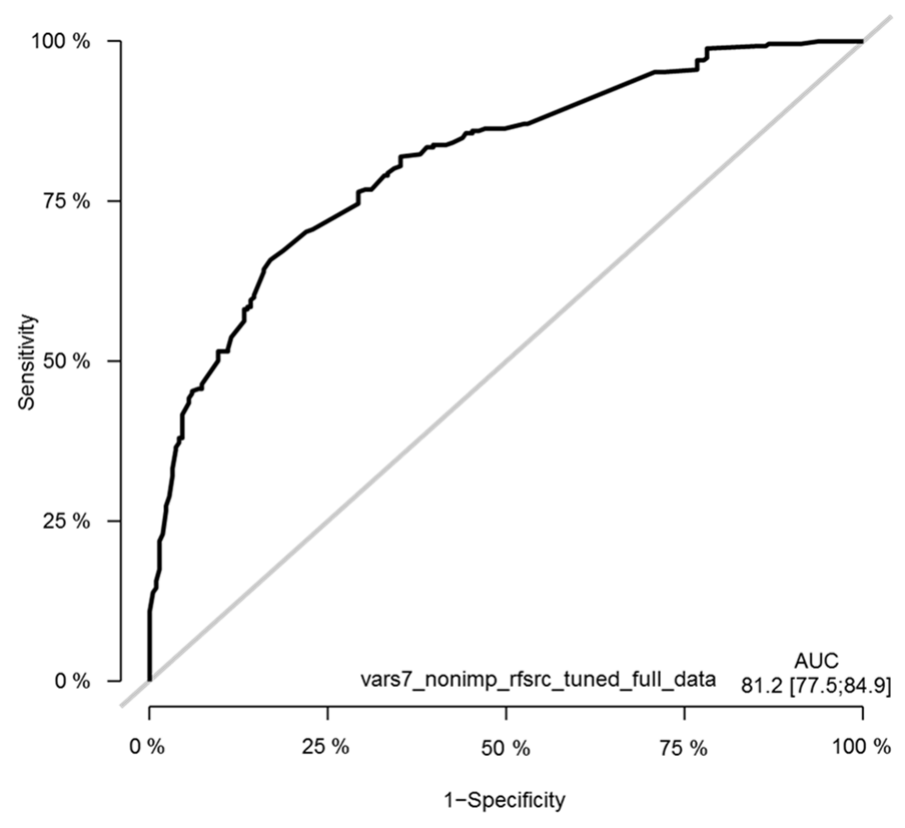

Figure 12. Time-dependent receiver operating characteristic (ROC) curves of the tuned seven-variable SRF model of the melanoma cohort. The ROC curve is shown at the timepoint of 36 months. The $y$-axis in the plot displays the true positive rate (TPR)—i.e., sensitivity—whereas the x-axis in the plot displays the false positive rate (FPR), i.e., specificity.

\subsection{The SRF-CLICAL Signature}

To validate the usage of the SRF model for predicting melanoma patient outcomes, the patients were divided into five risk groups-very high risk (Signature I), high risk (Signature II), medium risk (Signature III), low risk (Signature IV), and very low risk (Signature V) - based on their SRF-predicted survival probabilities. Survival curves for these five signatures were then compared (Figure 13). The five risk groups showed clearly and statistically significantly distinct survival curve profiles $(p<0.0001)$. These results are consistent, and validate the original simplified CLICAL signatures definition as shown in Figure 8.
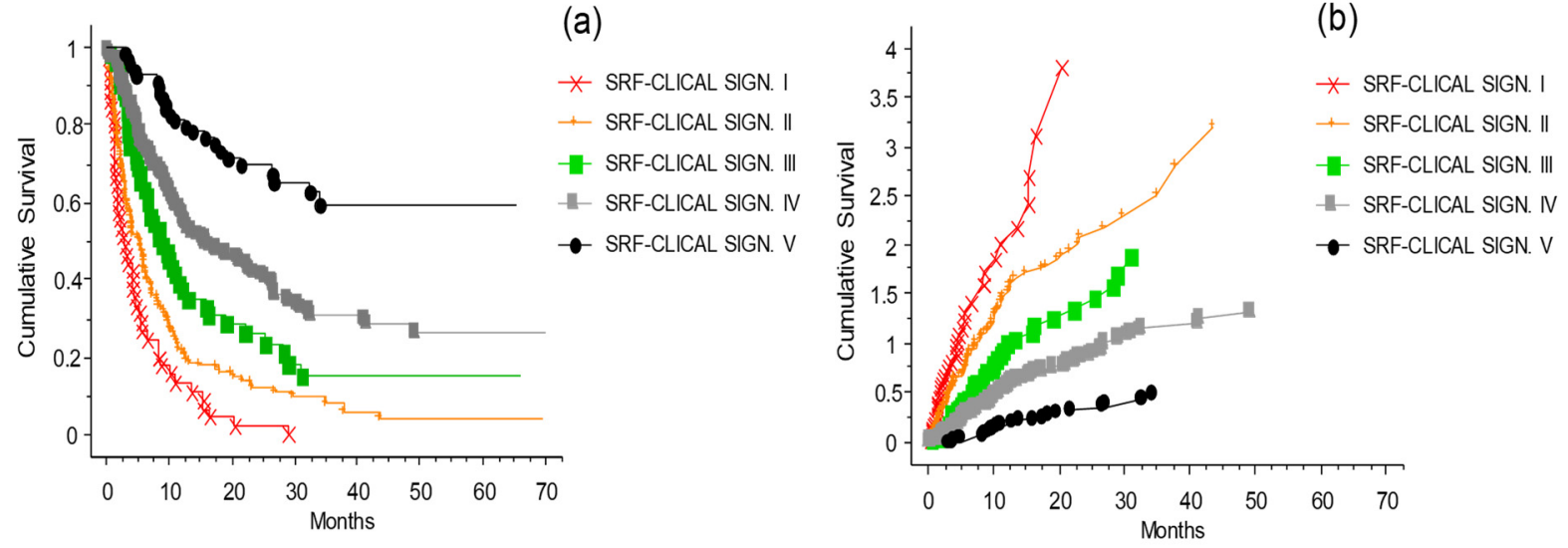

Figure 13. Kaplan-Meier survival curves of the melanoma patients divided into five risk groups based on their survival probability predictions obtained using the optimized survival random forest model. Plot (a) shows the cumulative survival with the distribution of the patients grouped by their signatures (applied to the risk groups defined by the SRF-CLICAL). The five curves are significantly separated from one another $(p<0.0001)$. (b) The presentation of the cumulative risks for each signature easily shows whether the characteristics of each signature are favorable or not. 


\section{Discussion}

Cutaneous melanoma is the most aggressive form of skin tumor, and its incidence has significantly increased in recent decades [29]. Fortunately, over the past few years, the development of immunotherapy with ICIs and TT against kinases of the RAS/BRAF/MAPK pathway has dramatically improved its clinical outcomes, with the achievement of longterm benefit in approximately $50 \%$ of patients with metastatic disease, completely changing the perspective for melanoma patients [30-32]. Immunotherapy has played a primary role due to the availability of new monoclonal antibodies directed toward the checkpoint molecules CTLA-4 and PD-1 [7]. The anti-CTLA-4 antibody (ipilimumab) can induce a response rate of approximately $15 \%$, with approximately $20 \%$ of patients being longterm responders [32]. The anti-PD-1 drugs (nivolumab or pembrolizumab) have shown a higher response rate of approximately $40 \%$ in treatment-naïve patients, with the majority of responses being durable [33]. However, the main limitations affecting the use of these agents are represented by the heterogeneous response of patients, and by the absence of universally recognized predictive biomarkers of response [34,35]. Based on these observations, in this study we have analyzed real-world data related to cutaneous metastatic melanoma patients treated with ICIs. To provide a useful tool for helping clinicians to make the best therapeutic decisions, we built an algorithm including patients' baseline clinical characteristics, investigating whether it could predict the response to treatment.

We retrospectively investigated 578 metastatic melanoma patients who received ipilimumab $(51 \%)$, pembrolizumab $(23 \%)$, or nivolumab $(26 \%)$ as monotherapies at the INT-NA. The whole cohort of 578 cases analyzed had an OS of $23 \%$ at 70 months (median 10 months; CI 95\%: 8.4-11.2); meanwhile, analyzing patients grouped by treatment received at the INT-NA, ipilimumab-treated patients had an OS of 15\% at 60 months (median 8.9 months; CI 95\%: 7.1-10.2), nivolumab-treated patients had an OS of $29.4 \%$ at 60 months (median 15.7 months; CI 95\%: 9.5-26.5), and pembrolizumab-treated patients had an OS of $25 \%$ at 60 months (median 11 months; CI 95\%: 7.2-16.2). Our results are consistent with clinical efficacy evidence derived from other real-life studies $[6,22,31,32,34]$. Moreover, we defined five groups of patients based on the therapy received before treatment at the INT-NA: $24 \%$ were naïve, $25 \%$ received immunotherapy, $18 \%$ received TT, $13 \%$ were treated with cytostatic agents, and 10\% received both TT and immunotherapy. Among these five groups, naïve and immune-pretreated patients had the highest survival, while patients pretreated with TT had the worst outcomes. Furthermore, data from other real-life retrospective analyses confirm our observations $[22,36,37]$. We proceeded to refine the analysis by grouping patients according to whether they had previously received TT or not. The group of patients who received TT responded poorly to the ICI treatments, and the effect of anti-PD-1 was not significantly different from that of anti-CTLA-4. In fact, patients who did not receive previous target treatment had more favorable outcomes in both cases. In addition, we analyzed the effect of target treatment on immunotherapy based on whether it was delivered before or after ICI treatment. The patients treated with TT after challenge with ICIs had a better OS compared to patients treated with ICIs after TT. Moreover, increasing evidence from the literature seems to be in accordance with our results. However, the most effective sequence of these agents has not been well characterized, although several studies were conducted to help make the best decisions for our patients [37-39].

Afterwards, the clinical variables routinely used to define the general status of disease for metastatic melanoma were analyzed for their risk of death or relapse [16,22,40-42]. Once we analyzed all of the clinical parameters, we could define and validate the prediction algorithm CLICAL which, based on the different score and relative signature attributed to each patient, is able to determine the degree of benefit obtained with ICI treatment for metastatic melanoma patients (Figure 8 and Table 4). This study shows that there is a group of patients where Signature V predicts an excellent response to the treatment, wherein more than $50 \%$ of the patients would still be alive at 70 months, while at the opposite end there is a group of patients where Signature I predicts no response. It should be noted that CLICAL Signatures IV and V include patients with high probability of survival regardless 
of the type of ICI. This means that patients treated with anti-CTLA-4 or anti-PD- 1 who have the highest scores have the same opportunity of response. On the other hand, the lowest signatures (I and II), regardless of the ICI, predict a very low or no benefit at all. This discriminant model raises the question of whether having this knowledge justifies commencing a treatment with the intention of obtaining lasting clinical benefit. To validate the potential prognostic role of the CLICAL, the algorithm was applied to an external cohort of 117 patients recruited at Karolinska University Hospital, Sweden. In this case, the CLICAL also identified different groups of patients, depending on outcome, with the same efficiency as observed in the INT-NA cohort. The use of signatures to determine categorical variables is increasing [20,43-45]. The clear distinction of the input and quality of information is relevant (patient's clinical variable, intervention, and time variable). Lastly, we introduced the machine learning SRF method to ensure that the CLICAL could identify a signature with high predictive power. The SRF-derived signature correlates to and visualizes the group selected by the original CLICAL empirical algorithm [23]. This knowledge might change future approaches to determining who to treat. In clinical praxis, this could also be applied in prospective clinical studies considering known validated variables for potential inclusion in the algorithm, and could predict the outcome of the individual patient depending on the defined signature at the beginning of ICI treatment.

\section{Conclusions}

In conclusion, with all of the limitations of a retrospective population-based study, we provide evidence that the analysis of real-life treatment of metastatic malignant melanoma patients reveals the possibility to increase the OS with ICI products. The collection of clinical parameters is an important tool in the analysis of their predictive power. In fact, this study shows that the application of the CLICAL and SRF-CLICAL algorithms can characterize individual patients with different benefits from ICI treatment. These prediction algorithms are likely to be useful for decision-making on ICI referrals, and to facilitate decisions on the eligibility of each patient with metastatic melanoma to enter the ICI program at the INT-NA. From the results of this study, we can ensure that the right patient receives the right treatment, which will benefit both the individual patient as well as the decision-making doctor.

Author Contributions: Conceptualization, G.M., G.V.M. and P.A.A.; data curation, G.M. and G.V.M.; formal analysis, G.V.M. and F.S.; funding acquisition, G.V.M. and P.A.A.; investigation, G.M., G.V.M., M.C., A.M.G., E.S., V.V., L.F. and P.A.A.; methodology, G.M., G.V.M., M.C., D.M., A.M.G., E.S., V.V., L.F., M.P., L.S., M.T., G.D. and P.A.A.; project administration, G.V.M.; resources, G.M., G.V.M. and F.S.; software, G.M., G.V.M. and F.S.; supervision, G.V.M. and P.A.A.; validation, L.V., I.K., H.E. and R.L.; writing — original draft preparation, G.M. and G.V.M.; writing—review and editing, G.M., G.V.M., M.C., D.M., A.M.G., E.S., V.V., L.F., M.P., L.S., M.T., G.D., L.V., I.K., H.E., F.S., R.L. and P.A.A. All authors have read and agreed to the published version of the manuscript.

Funding: This research was funded by the Italian Ministry of Health (IT-MOH) through "Ricerca Corrente", grant number M2-2. Additional funding (N\#184093) was obtained from the Stockholm Cancer Society and King Gustav V's Jubilee foundation Stockholm.

Institutional Review Board Statement: This study was conducted in accordance with the guidelines of the Declaration of Helsinki, and approved by the Institutional Review Board of the Istituto Nazionale Tumori IRCCS Fondazione "G. Pascale" (Naples, Italy) (protocol code 33/17oss, approved on 10 January 2018).

Informed Consent Statement: Informed consent was obtained from all subjects involved in the study.

Data Availability Statement: The datasets used and/or analyzed during the current study are available from the corresponding author on reasonable request. 
Conflicts of Interest: P.A.A. has/had consultant/advisory roles for Bristol Myers Squibb, RocheGenentech, Merck Sharp \& Dohme, Novartis, Array, Merck Serono, Pierre-Fabre, Incyte, Medimmune, AstraZeneca, Syndax, Sun Pharma, Sanofi, Idera, Ultimovacs, Sandoz, Im-munocore, 4SC, Alkermes, Italfarmaco, Nektar, Boehringer-Ingelheim, Eisai, Regeneron, Daiichi Sankyo, Pfizer, Oncosec, Nouscom, Takis, and Lunaphore; he also received research funding from Bristol Myers Squibb, Roche-Genentech, Array, and Sanofi, as well as travel support from MSD; all of the above took place outside the submitted work. A.M.G. has/had consultant/advisory roles for Bristol Myers Squibb, Merck Sharp \& Dohme, and Novartis; he also received travel grants from Bristol Myers Squibb, Merck Sharp \& Dohme, Novartis, Merck Serono, and Sun Pharma; all of the above took place outside the submitted work. All other authors declare no conflict of interest.

\section{References}

1. Ascierto, P.A.; Marincola, F.M. What have we learned from cancer immunotherapy in the last 3 years? J. Transl. Med. 2014, $12,141$. [CrossRef] [PubMed]

2. Schadendorf, D.; Dummer, R.; Hauschild, A.; Robert, C.; Hamid, O.; Daud, A.; Eertwegh, A.V.D.; Cranmer, L.; O’Day, S.; Puzanov, I.; et al. Health-related quality of life in the randomised KEYNOTE-002 study of pembrolizumab versus chemotherapy in patients with ipilimumab-refractory melanoma. Eur. J. Cancer 2016, 67, 46-54. [CrossRef] [PubMed]

3. Schadendorf, D.; Larkin, J.; Wolchok, J.; Hodi, F.S.; Sileni, V.C.; Gonzalez, R.; Rutkowski, P.; Grob, J.-J.; Cowey, C.L.; Lao, C.; et al. Health-related quality of life results from the phase III CheckMate 067 study. Eur. J. Cancer 2017, 82, 80-91. [CrossRef]

4. Petrella, T.M.; Robert, C.; Richtig, E.; Miller, W.H.; Masucci, G.V.; Walpole, E.; Lebbe, C.; Steven, N.; Middleton, M.R.; Hille, D.; et al. Patient-reported outcomes in KEYNOTE-006, a randomised study of pembrolizumab versus ipilimumab in patients with advanced melanoma. Eur. J. Cancer 2017, 86, 115-124. [CrossRef] [PubMed]

5. Mamoor, M.; Postow, M.A.; A Lavery, J.; Baxi, S.S.; Khan, N.; Mao, J.J.; Rogak, L.J.; Sidlow, R.; Thom, B.; Wolchok, J.A.; et al. Quality of life in long-term survivors of advanced melanoma treated with checkpoint inhibitors. J. Immunother. Cancer 2019, 8, e000260. [CrossRef]

6. Rogiers, A.; Boekhout, A.; Schwarze, J.K.; Awada, G.; Blank, C.U.; Neyns, B. Long-Term Survival, Quality of Life, and Psychosocial Outcomes in Advanced Melanoma Patients Treated with Immune Checkpoint Inhibitors. J. Oncol. 2019, 2019, 1-17. [CrossRef] [PubMed]

7. Madonna, G.; Ballesteros-Merino, C.; Feng, Z.; Bifulco, C.; Capone, M.; Giannarelli, D.; Mallardo, D.; Simeone, E.; Grimaldi, A.M.; Caracò, C.; et al. PD-L1 expression with immune-infiltrate evaluation and outcome prediction in melanoma patients treated with ipilimumab. OncoImmunology 2018, 7, e1405206. [CrossRef] [PubMed]

8. Pardoll, D.M. The blockade of immune checkpoints in cancer immunotherapy. Nat. Rev. Cancer 2012, 12, 252-264. [CrossRef] [PubMed]

9. Hodi, F.S.; O’Day, S.J.; McDermott, D.F.; Weber, R.W.; Sosman, J.A.; Haanen, J.B.; Gonzalez, R.; Robert, C.; Schadendorf, D.; Hassel, J.C.; et al. Improved Survival with Ipilimumab in Patients with Metastatic Melanoma. N. Engl. J. Med. 2010, 363, 711-723. [CrossRef] [PubMed]

10. Larkin, J.; Sileni, V.C.; Gonzalez, R.; Grob, J.-J.; Cowey, C.L.; Lao, C.D.; Schadendorf, D.; Dummer, R.; Smylie, M.; Rutkowski, P.; et al. Combined Nivolumab and Ipilimumab or Monotherapy in Untreated Melanoma. N. Engl. J. Med. 2015, 373, 23-34. [CrossRef] [PubMed]

11. Balar, A.; Weber, J.S. PD-1 and PD-L1 antibodies in cancer: Current status and future directions. Cancer Immunol. Immunother. 2017, 66, 551-564. [CrossRef]

12. Larkin, J.; Minor, D.; D’Angelo, S.; Neyns, B.; Smylie, M., Jr.; Gutzmer, R.; Linette, G.; Chmielowski, B.; Lao, C.D. Overall Survival in Patients with Advanced Melanoma Who Received Nivolumab Versus Investigator's Choice Chemotherapy in CheckMate 037: A Randomized, Controlled, Open-Label Phase III Trial. J. Clin. Oncol. 2018, 36, 383-390. [CrossRef] [PubMed]

13. Schachter, J.; Ribas, A.; Long, G.; Arance, A.; Grob, J.-J.; Mortier, L.; Daud, A.; Carlino, M.S.; McNeil, C.; Lotem, M.; et al. Pembrolizumab versus ipilimumab for advanced melanoma: Final overall survival results of a multicentre, randomised, openlabel phase 3 study (KEYNOTE-006). Lancet 2017, 390, 1853-1862. [CrossRef]

14. Harder, N.; Schönmeyer, R.; Nekolla, K.; Meier, A.; Brieu, N.; Vanegas, C.; Madonna, G.; Capone, M.; Botti, G.; Ascierto, P.A.; et al. Automatic discovery of image-based signatures for ipilimumab response prediction in malignant melanoma. Sci. Rep. 2019, 9, 1-19. [CrossRef]

15. Capone, M.; Fratangelo, F.; Giannarelli, D.; Sorrentino, C.; Turiello, R.; Zanotta, S.; Galati, D.; Madonna, G.; Tuffanelli, M.; Scarpato, L.; et al. Frequency of circulating CD8+CD73+T cells is associated with survival in nivolumab-treated melanoma patients. J. Transl. Med. 2020, 18, 1-10. [CrossRef] [PubMed]

16. Schilling, B.; Martens, A.; Foppen, M.H.G.; Gebhardt, C.; Hassel, J.C.; Rozeman, E.A.; Gesierich, A.; Gutzmer, R.; Kähler, K.C.; Livingstone, E.; et al. First-line therapy-stratified survival in BRAF-mutant melanoma: A retrospective multicenter analysis. Cancer Immunol. Immunother. 2019, 68, 765-772. [CrossRef]

17. Ayers, M.; Lunceford, J.; Nebozhyn, M.; Murphy, E.; Loboda, A.; Kaufman, D.R.; Albright, A.; Cheng, J.D.; Kang, S.P.; Shankaran, V.; et al. IFN- $\gamma$-related mRNA profile predicts clinical response to PD-1 blockade. J. Clin. Investig. 2017, 127, 2930-2940. [CrossRef] [PubMed] 
18. Tucci, M.; Passarelli, A.; Mannavola, F.; Stucci, L.S.; Ascierto, P.A.; Capone, M.; Madonna, G.; Lopalco, P.; Silvestris, F. Serum exosomes as predictors of clinical response to ipilimumab in metastatic melanoma. OncoImmunology 2017, 7, e1387706. [CrossRef]

19. Jacquelot, N.; Yamazaki, T.; Roberti, M.P.; Duong, C.P.M.; Andrews, M.C.; Verlingue, L.; Ferrere, G.; Becharef, S.; Vétizou, M.; Daillère, R.; et al. Sustained Type I interferon signaling as a mechanism of resistance to PD-1 blockade. Cell Res. $2019,29,846-861$. [CrossRef] [PubMed]

20. Madonna, G.; Capone, M.; Tuffanelli, M.; Curvietto, M.; Paone, M.; Esposito, A.; Sorrentino, A.; Palla, M.; Scarpato, L.; Mallardo, D.; et al. Real World data analysis related to metastatic melanoma patients treated with immunotherapy from 2012 to 2018 at Istituto Nazionale Tumori IRCCS Fondazione "G. Pascale" of Napoli, Italy. 34th Annual Meeting \& Pre-Conference Programs of the Society for Immunotherapy of Cancer (SITC 2019): Part 1. J. Immunother. Cancer 2019, 7, 272-282. [CrossRef]

21. Wolchok, J.D.; Hoos, A.; O’Day, S.; Weber, J.; Hamid, O.; Lebbé, C.; Maio, M.; Binder, M.; Bohnsack, O.; Nichol, G.; et al. Guidelines for the Evaluation of Immune Therapy Activity in Solid Tumors: Immune-Related Response Criteria. Clin. Cancer Res. 2009, 15, 7412-7420. [CrossRef]

22. Krakowski, I.; Bottai, M.; Häbel, H.; Masucci, G.; Girnita, A.; Smedby, K.; Eriksson, H. Impact of modern systemic therapies and clinical markers on treatment outcome for metastatic melanoma in a real-world setting. J. Eur. Acad. Dermatol. Venereol. 2020, 35, 105-115. [CrossRef]

23. Simao, F.A.; Hieta, R.; Pulman, J.A.; Madonna, G.; Ascierto, P.A.; Lewensohn, R.; Masucci, G.V. SRF-CLICAL: An approach for patient risk stratification using random forest models. bioRxiv 2021. [CrossRef]

24. Therneau, T. A Package for Survival Analysis in R, Version 3.2-3. 2020. Available online: https: / CRAN.R-project.org/package= survival (accessed on 13 March 2021).

25. Kassambara, A.; Kosinski, M. Survminer: Drawing Survival Curves Using 'ggplot2' R Package Version 0.4.8. 2018. Available online: https: / /CRAN.R-project.org/package=survminer (accessed on 9 March 2021).

26. Mogensen, U.B.; Ishwaran, H.; Gerds, T.A. Evaluating Random Forests for Survival Analysis Using Prediction Error Curves. J. Stat. Softw. 2012, 50, 1-23. Available online: http:/ / www.jstatsoft.org/v50/i11/ (accessed on 18 September 2020). [CrossRef]

27. Gerds, T.A.; Ozenne, B. Risk Regression: Risk Regression Models and Prediction Scores for Survival Analysis with Competing Risks. R Package Version 2020.02.05. 2020. Available online: https:/ / CRAN.R-project.org/package=riskRegression (accessed on 9 December 2020).

28. Ishwaran, H.; Kogalur, U.B. Fast Unified Random Forests for Survival, Regression, and Classification (RF-SRC), R Package Version 2.9.3. 2021. Available online: https:// cran.r-project.org/package=randomForestSRC (accessed on 9 March 2021).

29. Ruggiero, C.F.; Malpicci, D.; Fattore, L.; Madonna, G.; Vanella, V.; Mallardo, D.; Liguoro, D.; Salvati, V.; Capone, M.; Bedogni, B.; et al. ErbB3 Phosphorylation as Central Event in Adaptive Resistance to Targeted Therapy in Metastatic Melanoma: Early Detection in CTCs during Therapy and Insights into Regulation by Autocrine Neuregulin. Cancers 2019, 11, 1425. [CrossRef]

30. Villani, A.; Scalvenzi, M.; Fabbrocini, G.; Ocampo-Candiani, J.; Ocampo-Garza, S.S. Looking into a Better Future: Novel Therapies for Metastatic Melanoma. Dermatol. Ther. 2021, 11, 751-767. [CrossRef]

31. Luke, J.J.; Flaherty, K.T.; Ribas, A.; Long, G. Targeted agents and immunotherapies: Optimizing outcomes in melanoma. Nat. Rev. Clin. Oncol. 2017, 14, 463-482. [CrossRef] [PubMed]

32. Mason, R.; Au, L.; Garces, A.I.R.; Larkin, J. Current and emerging systemic therapies for cutaneous metastatic melanoma. Expert Opin. Pharmacother. 2019, 20, 1135-1152. [CrossRef]

33. Guida, M.; Bartolomeo, N.; Quaglino, P.; Madonna, G.; Pigozzo, J.; Di Giacomo, A.; Minisini, A.; Tucci, M.; Spagnolo, F.; Occelli, M.; et al. No Impact of NRAS Mutation on Features of Primary and Metastatic Melanoma or on Outcomes of Checkpoint Inhibitor Immunotherapy: An Italian Melanoma Intergroup (IMI) Study. Cancers 2021, 13, 475. [CrossRef] [PubMed]

34. Ottaviano, M.; De Placido, S.; Ascierto, P.A. Recent success and limitations of immune checkpoint inhibitors for cancer: A lesson from melanoma. Virchows Arch. 2019, 474, 421-432. [CrossRef] [PubMed]

35. Bedognetti, D.; Ceccarelli, M.; Galluzzi, L.; Lu, R.; Palucka, K.; Samayoa, J.; Spranger, S.; Warren, S.; Wong, K.K. Toward a comprehensive view of cancer immune responsiveness: A synopsis from the SITC workshop. J. Immunother. Cancer 2019, 7, 131. [CrossRef] [PubMed]

36. Donia, M.; Ellebaek, E.; Øllegaard, T.H.; Duval, L.; Aaby, J.B.; Hoejberg, L.; Køhler, U.H.; Schmidt, H.; Bastholt, L.; Svane, I.M. The real-world impact of modern treatments on the survival of patients with metastatic melanoma. Eur. J. Cancer 2019, 108, 25-32. [CrossRef] [PubMed]

37. Moser, J.C.; Chen, D.; Hu-Lieskovan, S.; Grossmann, K.F.; Patel, S.; Colonna, S.V.; Ying, J.; Hyngstrom, J.R. Real-world survival of patients with advanced BRAF V600 mutated melanoma treated with front-line BRAF/MEK inhibitors, anti-PD-1 antibodies, or nivolumab/ipilimumab. Cancer Med. 2019, 8, 7637-7643. [CrossRef]

38. Pavlick, A.C.; Fecher, L.; Ascierto, P.A.; Sullivan, R.J. Frontline Therapy for BRAF-Mutated Metastatic Melanoma: How Do You Choose, and Is There One Correct Answer? Am. Soc. Clin. Oncol. Educ. Book 2019, 39, 564-571. [CrossRef]

39. Johnson, D.B.; Pectasides, E.; Feld, E.; Ye, F.; Zhao, S.; Johnpulle, R.; Merritt, R.; McDermott, D.F.; Puzanov, I.; Lawrence, D.; et al. Sequencing Treatment in BRAF V600 Mutant Melanoma: Anti-PD-1 Before and After BRAF Inhibition. J. Immunother. 2017, 40, 31-35. [CrossRef] [PubMed]

40. Rozeman, E.A.; Dekker, T.; Haanen, J.B.A.G.; Blank, C.U. Advanced Melanoma: Current Treatment Options, Biomarkers, and Future Perspectives. Am. J. Clin. Dermatol. 2017, 19, 303-317. [CrossRef] 
41. Weide, B.; Martens, A.; Hassel, J.C.; Berking, C.; Postow, M.A.; Bisschop, K.; Simeone, E.; Mangana, J.; Schilling, B.; Di Giacomo, A.M.; et al. Baseline Biomarkers for Outcome of Melanoma Patients Treated with Pembrolizumab. Clin. Cancer Res. 2016, 22, 5487-5496. [CrossRef] [PubMed]

42. Zhao, B.; Zhao, H.; Zhao, J. Impact of clinicopathological characteristics on survival in patients treated with immune checkpoint inhibitors for metastatic melanoma. Int. J. Cancer 2018, 144, 169-177. [CrossRef] [PubMed]

43. Nie, R.-C.; Yuan, S.-Q.; Wang, Y.; Chen, Y.-B.; Cai, Y.-Y.; Chen, S.; Li, S.-M.; Zhou, J.; Chen, G.-M.; Luo, T.-Q.; et al. Robust immunoscore model to predict the response to anti-PD1 therapy in melanoma. Aging 2019, 11, 11576-11590. [CrossRef] [PubMed]

44. Liu, D.; Schilling, B.; Liu, D.; Sucker, A.; Livingstone, E.; Jerby-Arnon, L.; Zimmer, L.; Gutzmer, R.; Satzger, I.; Loquai, C.; et al. Author Correction: Integrative molecular and clinical modeling of clinical outcomes to PD1 blockade in patients with metastatic melanoma. Nat. Med. 2020, 26, 1147. [CrossRef]

45. Danaher, P.; Warren, S.; Lu, R.; Samayoa, J.; Sullivan, A.; Pekker, I.; Wallden, B.; Marincola, F.M.; Cesano, A. Pan-cancer adaptive immune resistance as defined by the Tumor Inflammation Signature (TIS): Results from The Cancer Genome Atlas (TCGA). J. Immunother. Cancer 2018, 6, 63. [CrossRef] [PubMed] 